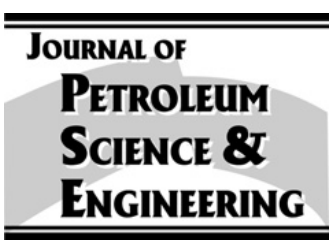

www.elsevier.com/locate/petrol

\title{
Pore-scale network modeling of Ellis and Herschel-Bulkley fluids
}

\author{
Taha Sochi*, Martin J. Blunt \\ Department of Earth Science and Engineering, Imperial College London, Prince Consort Road, SW7 2AZ, United Kingdom
}

Received 20 October 2006; accepted 26 May 2007

\begin{abstract}
Network modeling is used to study the flow in porous media of Ellis and Herschel-Bulkley fluids, which model a large group of time-independent non-Newtonian fluids. Previous work is extended to include yield-stress and shear-thickening phenomena. We use two topologically-disordered networks representing a sand pack and Berea sandstone. Analytical expressions for the volumetric flow rate in a single tube are derived and implemented in each pore and throat to simulate single-phase flow in the pore space. An iterative technique is used to compute the relationship between flow rate and pressure gradient across the whole network. The single tube behavior is compared to that of the network. Experimental data in the literature are compared to the network simulation results to validate the model and investigate its predictive capabilities. Good agreement is obtained in many cases. The flow of yield-stress fluids in porous media is analyzed. Two algorithms to predict the network threshold yield pressure are implemented and compared to the numerically computed threshold yield pressure of the network.
\end{abstract}

(C) 2007 Elsevier B.V. All rights reserved.

Keywords: Ellis; Herschel-Bulkley; Non-Newtonian fluid; Bingham plastic; Shear thinning; Shear thickening; Yield stress; Network modeling; Pore-scale; Porous media

\section{Introduction}

The study of the flow of non-Newtonian fluids in porous media has a wide variety of practical applications in processes such as enhanced oil recovery from underground reservoirs, filtration of polymer solutions and soil remediation through the removal of liquid pollutants. Heavy oils, foams, polymer solutions and viscoelastic surfactants are some non-Newtonian fluids that are commonly found in porous media in oil field settings.

Despite a large number of studies on non-Newtonian flow in porous media (Sadowski and Bird, 1965;

\footnotetext{
* Corresponding author.

E-mail addresses: taha.sochi@imperial.ac.uk (T. Sochi), m.blunt@imperial.ac.uk (M.J. Blunt).
}

Gaitonde and Middleman, 1966; Marshall and Metzner, 1966; Kozicki et al., 1967; Dauben and Menzie, 1967; White, 1967; Dominguez and Willhite, 1977; Alvarado and Marsden, 1979; Sorbie, 1991), it is still not possible, reliably, to relate the porous medium structure and bulk rheology of the fluid to the in-situ flow behavior. For shear-thinning fluids, it is possible to define an apparent shear rate in the porous medium and to use this to predict the apparent viscosity (Sorbie, 1991; Pearson and Tardy, 2002). However, to make quantitative predictions, it is necessary to invoke an empirical factor that depends on the fluid and rock properties (Sorbie, 1991; Pearson and Tardy, 2002). If the fluids have a yield stress, there is the further complication of defining the pressure gradient at which flow first starts.

One successful approach to predicting flow in porous media is network modeling (Blunt, 2001; Øren et al., 
1998). The pore space is represented by a lattice of pores connected by throats. Then, once the flow behavior in a single pore or throat is known, the overall flow in the network can be computed. In recent years, the combination of realistic representations of the pore space and accurate models of pore-level physics has allowed predictions of a variety of properties, such as relative permeability, capillary pressure and dispersion coefficient to be made (Øren et al., 1998; Blunt et al., 2002; Valvatne and Blunt, 2004; Bijeljic et al., 2004; Piri and Blunt, 2005).

Sorbie and co-workers developed a network model of shear-thinning fluids and included the effects of sorption and excluded volume (Sorbie et al., 1987; Sorbie, 1989, 1991; Sorbie and Clifford, 1989, 1991; Huang and Sorbie, 1992). Other authors have also studied nonNewtonian flow in network models (Tsakiroglou, 2002, 2004; Fadili et al., 2002; Tsakiroglou et al., 2003; Balhoff and Thompson, 2004, 2006; Balhoff, 2004; Chen et al., 2004, 2005a,b). Most of these groups used regular lattices and so could not make quantitative comparisons with experimental measurements in rock samples. Lopez et al. (2003) developed a pore network model of shear-thinning fluids. Using an accurate characterization of the bulk rheology and realistic networks they were able to predict the results of several experimental data sets in the literature. Balhoff and Thompson (2004), and Balhoff (2005) studied the steady flow of yield-stress fluids using computer-generated random sphere packings as models of packed beds. They later (Balhoff, 2004; Balhoff and Thompson, 2006) investigated shear-thinning power-law and Ellis fluids using a similar approach. The model was used to predict experimental measurements for Bingham fluids. Chen et al. (2004, 2005a,b) performed a detailed pore-level analysis of foam that also acts as a yield-stress fluid and discussed the onset of flow as the pressure gradient increased.

In this paper, we will extend the work of Lopez et al. to include Ellis and Herschel-Bulkley models. The advantage of studying Ellis fluids is the availability of an analytical expression for the volumetric flow rate in a single throat. Moreover, unlike the power-law model it demonstrates a realistic viscous behavior at low-shear. The advantage of using the Herschel-Bulkley model is that, besides the availability of an analytical expression for the flow rate, it can model all the main classes of the time-independent flow behavior including shear-thickening and yield-stress fluids. We will first discuss the porous medium behavior as a function of fluid properties and pore structure before predicting experimental data in the literature. We will also investigate the behavior of yield-stress fluids in porous media.

\section{Bulk rheology of Ellis and Herschel-Bulkley fluids}

\subsection{Ellis model}

This is a three-parameter model which describes timeindependent shear-thinning yield-free non-Newtonian fluids. Its distinctive feature is the low-shear Newtonian plateau without a high-shear one. According to this model, the fluid viscosity $\mu$ is given by (Sadowski and Bird, 1965; Savins, 1969; Bird et al., 1987; Carreau et al., 1997):

$\mu=\frac{\mu_{0}}{1+\left(\frac{\tau}{\tau_{1 / 2}}\right)^{\alpha-1}}$

where $\mu_{0}$ is the low-shear viscosity, $\tau$ is the shear stress, $\tau_{1 / 2}$ is the shear stress at which $\mu=\mu_{0} / 2$ and $\alpha$ is an indicial parameter.

For Ellis fluids, the volumetric flow rate in circular cylindrical tube is given by (Sadowski and Bird, 1965; Savins, 1969; Bird et al., 1987; Carreau et al., 1997):

$Q=\frac{\pi R^{4} \Delta P}{8 L \mu_{0}}\left[1+\frac{4}{\alpha+3}\left(\frac{R \Delta P}{2 L \tau_{1 / 2}}\right)^{\alpha-1}\right]$

where $\mu_{0}, \tau_{1 / 2}$ and $\alpha$ are the Ellis parameters, $R$ is the tube radius, $\Delta P$ is the pressure drop across the tube and $L$ is the tube length. The derivation of this expression can be found in Appendix A.

\subsection{Herschel-Bulkley model}

The Herschel-Bulkley model has three parameters and can describe Newtonian and a large group of timeindependent non-Newtonian fluids. It is given by (Skelland, 1967):

$\tau=\tau_{\mathrm{o}}+C \gamma^{n}\left(\tau>\tau_{\mathrm{o}}\right)$

where $\tau$ is the shear stress, $\tau_{\mathrm{o}}$ is the yield stress above which the fluid starts flowing, $C$ is the consistency factor, $\gamma$ is the shear rate and $n$ is the flow behavior index.

There are six main classes to this model: shear thinning $(n<1.0)$, shear thickening $(n>1.0)$ and neither $(n=1.0)$; each either with yield stress $\left(\tau_{\mathrm{o}}>0\right)$ or without yield stress $\left(\tau_{\mathrm{o}}>0\right)$. For the determination of rheological parameters for Herschel-Bulkley fluids, the interested reader is referred to Kelessidis et al. (2006). 
For Herschel-Bulkley fluids, the volumetric flow rate in a circular cylindrical tube is given by (Skelland, 1967):

$$
\begin{aligned}
Q= & \frac{8 \pi}{C^{1}}\left(\frac{L}{\Delta P}\right)^{3}\left(\tau_{\mathrm{w}}-\tau_{\mathrm{o}}\right)^{1+\frac{1}{n}} \\
& \times\left[\frac{\left(\tau_{\mathrm{w}}-\tau_{\mathrm{o}}\right)^{2}}{3+1 / n}+\frac{2 \tau_{\mathrm{o}}\left(\tau_{\mathrm{w}}-\tau_{\mathrm{o}}\right)}{2+1 / n}+\frac{\tau_{\mathrm{o}}^{2}}{1+1 / n}\right]\left(\tau_{\mathrm{w}}>\tau_{\mathrm{o}}\right)
\end{aligned}
$$

where $\tau_{\mathrm{o}}, C$ and $n$ are the Herschel-Bulkley parameters, $L$ is the tube length, $\Delta P$ is the pressure drop across the tube and $\tau_{\mathrm{w}}$ is the shear stress at the tube wall $(=\Delta P R / 2 L)$. The derivation of this expression can be found in Appendix A.

For yield-stress fluids, the threshold pressure drop, $\Delta P_{\text {th }}$, above which the flow in a single tube starts, is:

$\Delta P_{\mathrm{th}}=\frac{2 L \tau_{\mathrm{o}}}{R}$.

This can be obtained from the fact that for a HerschelBulkley fluid with a yield stress the flow occurs when $Q>0$ where $Q$ is given by Eq. (4). Assuming $\tau_{\mathrm{o}}, R, L$, $C, \Delta P, n>0$, it is straightforward to show that the condition $Q>0$ is satisfied iff $\left(\tau_{\mathrm{w}}-\tau_{\mathrm{o}}\right)>0$, that is:

$\tau_{\mathrm{w}}=\frac{\Delta P R}{2 L}>\tau_{\mathrm{o}}$

which produces Eq. (5). Alternatively, the flow occurs when the shear stress at the tube wall exceeds the yield stress, i.e. $\tau_{\mathrm{w}}>\tau_{\mathrm{o}}$, which leads to the same condition. The second argument is less obvious but more general than the first. Characterization of the flow regime in pipes for yield-stress fluids is investigated by Gücüyener and Mehmetoğlu (1996).

\section{Previous work}

Here we outline some advances in the investigation of the flow of Ellis and Herschel-Bulkley fluids in porous media.

Sadowski and Bird (1965), and Sadowski (1963, 1965) applied the Ellis model to a non-Newtonian fluid in a porous medium modeled by a bundle of capillaries of varying cross-section and derived a generalized form of Darcy's law. They backed their theoretical investigation by experimental work. Park (1972), and Park et al. (1973) used the Ellis model as an alternative to a power-law form in modeling the flow of various aqueous polymeric solutions in packed beds of glass beads and experimentally validated their findings.
Balhoff and Thompson (2006), and Balhoff (2005) carried out experimental work on the flow of guar gum solution, modeled as an Ellis fluid, in packed beds of glass beads. The results of their network computer model matched the experimental data very well within an adjustable parameter.

The flow of Herschel-Bulkley and yield-stress fluids in porous media has been investigated by several authors. Park et al. (1973), and Park (1972) used the Ergun equation to correlate pressure drop to flow rate in packed beds by using a derived effective viscosity and validated their model by experimental work. To describe the non-steady flow of a yield-stress fluid in porous media, Pascal (1981) modified Darcy's law by introducing a threshold pressure gradient to account for the yield stress. This threshold gradient is directly proportional to the yield stress and inversely proportional to the square root of the absolute permeability. Al-Fariss and Pinder (1984), and Al-Fariss (1989) produced a general form of Darcy's law by modifying the Blake-Kozeny equation. They ended with very similar equations to those obtained by Pascal. Their work includes experimental investigation of the flow of waxy oils through packed beds of sand. Wu et al. (1992) applied an integral analytical method to obtain an approximate analytical solution for single-phase flow of Bingham fluids through porous media. They also developed a Buckley-Leverett analytical solution for 1D flow in porous media to study the displacement of a Bingham fluid by a Newtonian fluid. Chaplain et al. (1992) modeled the flow of a Bingham fluid through porous media by generalizing Saffman (1959) analysis for Newtonian flow to describe the dispersion in a porous medium by a random walk. Vradis and Protopapas (1993) extended the "capillary tube" and the "resistance to flow" models to describe the flow of Bingham fluids in porous media and presented a solution in which the flow is zero below a threshold head gradient and Darcian above it. They, like Pascal, analytically demonstrated that in both models the minimum pressure gradient required for the initiation of flow is proportional to the yield stress and inversely proportional to the characteristic length scale of the porous medium. Chase and Dachavijit (2003) modified the Ergun equation to describe the flow of yield-stress fluids through porous media by applying a bundle of capillary tubes approach similar to that of Al-Fariss. They also experimentally investigated the flow of Bingham aqueous solutions of Carbopol 941 through packed beds of glass beads. Recently, Balhoff and Thompson (2004), and Balhoff (2005) used their 3D network model based on computer-generated random sphere packing to investigate the flow of Bingham 
fluids in packed beds. To model non-Newtonian flow in the throats, they used new numerically-derived equations to accurately represent the throat geometry. The adjustments were made specifically for each individual fluid type. Our work will extend these studies to study the full range of Herschel-Bulkley behavior in porous media.

\section{Network modeling}

Our network model was originally developed by Valvatne and co-workers (Lopez et al., 2003; Valvatne and Blunt, 2004) and is modified and extended to include Herschel-Bulkley and Ellis models and to implement yield-stress algorithms.

The model uses 3D networks built from a topologically-equivalent $3 \mathrm{D}$ voxel image of the pore space with the pore sizes, shapes and connectivity reflecting the real medium. While there is some local spatial correlation of pore and throat size, at the scale of the whole network the systems are statistically homogeneous (Øren and Bakke, 2003). Pores and throats are modeled as having triangular, square or circular crosssection by assigning a shape factor which is the ratio of the area to the perimeter squared and obtained from the pore space image. Most of the network elements are not circular. To account for the non-circularity when calculating the volumetric flow rate from the analytical expression for a cylindrical tube, an equivalent radius $R_{\text {eq }}$ is defined:

$R_{\mathrm{eq}}=\left(\frac{8 G}{\pi}\right)^{1 / 4}$

where the conductance, $G$, is obtained empirically from numerical simulation.

Because initially the pressure drop in each network element is not known, an iterative method is used. This starts by assigning an effective viscosity, $\mu_{\text {eff }}$, to each network element. By invoking the conservation of volume for incompressible fluid, the pressure field across the entire network is solved by an algebraic multi-grid solver (Ruge and Stüben, 1987). Knowing the pressure drop, the effective viscosity of each element is updated using the analytical expression for the flow rate with a pseudo-Poiseuille definition. The pressure is then recomputed using the updated viscosities and the iteration continues until convergence is achieved when a specified tolerance error in total flow rate between two consecutive iteration cycles is reached. Finally, the total flow rate and the apparent viscosity are obtained.

\section{Network model results}

In this section we study generic trends in behavior for the Herschel-Bulkley model. We do not do this for the Ellis model, since its behavior as a shear-thinning fluid is included within the Herschel-Bulkley model and has already been studied previously (Lopez et al., 2003).

\subsection{Network-single tube comparison}

A comparison is made between a network representing a porous medium and a single tube of a bundle of capillaries of uniform radius all aligned with the flow direction. The radius of the single tube, $R$, is given by:

$R=\sqrt{\frac{8 K}{\phi}}$

where $K$ and $\phi$ are the respective absolute permeability and porosity of the network. This radius will be smaller than the average throat radius of a topologicallydisordered network, since the single tube has no tortuosity. This expression can be obtained by equating the Poiseuille linear flow rate for such a tube to the Newtonian Darcy velocity of the network assuming identical porosities.

Two networks representing two different porous media have been investigated: a sand pack and a Berea sandstone. In each case, two model fluids were studied: a fluid with no yield stress and a fluid with a yield stress. For each fluid, the flow behavior index, $n$, takes the values $0.6,0.8,1.0,1.2$ and 1.4. In all cases the consistency factor, $C$, is kept constant at $0.1 \mathrm{~Pa}$. $\mathrm{s}^{n}$, as it is considered a viscosity scale factor.

\subsubsection{Sand pack network}

The physical and statistical details of this network can be found in Appendix B (Table 7). The network represents a random close packing of similarly-sized grains and has relatively narrow pore and throat size distributions. The comparison between the sand pack network and single tube for the case of a fluid with no yield stress is displayed in Fig. 1. By definition, the network and single tube results are identical in the Newtonian case. In the porous medium the relationship between Darcy velocity, $q$, and pressure gradient in the flow direction, $\nabla P$, is given by:

$q=-\frac{K}{\mu_{\text {app }}}|\nabla P|$ 


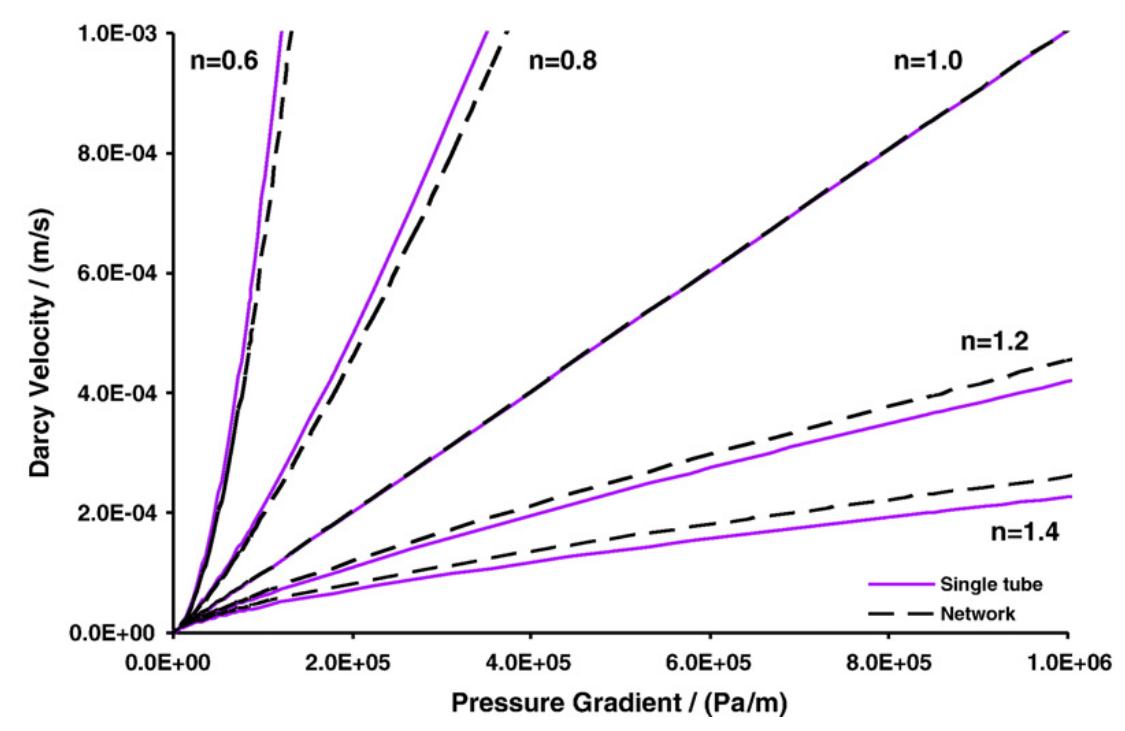

Fig. 1. Comparison between the sand pack network $\left(K=1.0 \times 10^{-10} \mathrm{~m}^{2}, \phi=0.35\right)$ and single tube $(R=48.2 \mu \mathrm{m})$ for a fluid with $\tau_{\mathrm{o}}=0.0$ Pa and $C=0.1 \mathrm{~Pa} . \mathrm{s}^{n}$.

where $\mu_{\text {app }}$ is the apparent viscosity of the fluid in the porous medium and $K$ is the absolute permeability. With no yield stress, $\mu_{\text {app }}$ can be related to the viscosity as a function of shear rate, $\mu(\gamma)$, measured in bulk by the following empirical relation (Cannella et al., 1988; Lopez et al., 2003):

$\mu_{\text {app }}(q)=\mu\left(\gamma=\alpha \frac{q}{\sqrt{K \phi}}\right)$

with a constant $\alpha$ that depends on the porous medium and fluid properties. For a single tube it can be shown that (Sorbie, 1991):

$\alpha=\sqrt{2}\left(\frac{3 n+1}{4 n}\right)$.

For $n=0.6, \alpha=1.65$ for a single tube while $\alpha=1.37$ for the network (Lopez et al., 2003), indicating that the apparent viscosity for a single tube is lower than the network, resulting in a higher Darcy velocity for a given pressure gradient, as seen. The same behavior is seen for all $n<1.0$. The sand pack network is relatively homogeneous and has a value of $\alpha$ close to 1.0.

For $n>1.0$ the situation is reversed. For $n=1.4$, $\alpha=1.31$ for a single tube. Again the apparent value of $\alpha$ for the network is lower than that for the tube. Since the medium is now shear-thickening, the apparent viscosity is now higher in the single tube, giving a lower Darcy velocity.

The comparison between the sand pack network and single tube for the case of a fluid with a yield stress is displayed in Fig. 2. The first thing to remark is that the sand pack network starts flowing at a lower pressure gradient than the single tube. Plotting a graph of the radius of the single tube and the average radius of the non-blocked throats of the network as a function of pressure gradient reveals that the average radius of the flowing throats at yield is slightly greater than the single tube radius as seen in Fig. 3. The average radius is only shown once there is a finite flow rate across the network. Eq. (5) implies that the corresponding pressure gradient is lower.

However, the average radius of flowing elements ultimately becomes smaller than the single tube. Hence for a Bingham fluid, the flow of the sand pack network exceeds the single tube flow at low pressure gradients, but the trend is reversed eventually. The reason is that the network yields before the single tube but since some network elements are blocked even at high pressure gradients, the single tube flow will eventually exceed the network flow.

For the shear-thinning cases, the prominent feature is the crossover between the network and single tube curves. This is due to the shift in the relation between the average radius of the non-blocked elements and the radius of the single tube as seen in Fig. 3. The intersect for $n=0.6$ occurs at higher pressure gradient than that for $n=0.8$ because of the flow enhancement in the network, which yields first, caused by more shearthinning in the $n=0.6$ case. This enhancement delays the catchup of the single tube to a higher pressure gradient.

For the shear-thickening cases, the situation is more complex. There are three main factors affecting the network-single tube relation: the partial blocking of the network, the shift in the average tube radius relation, and 


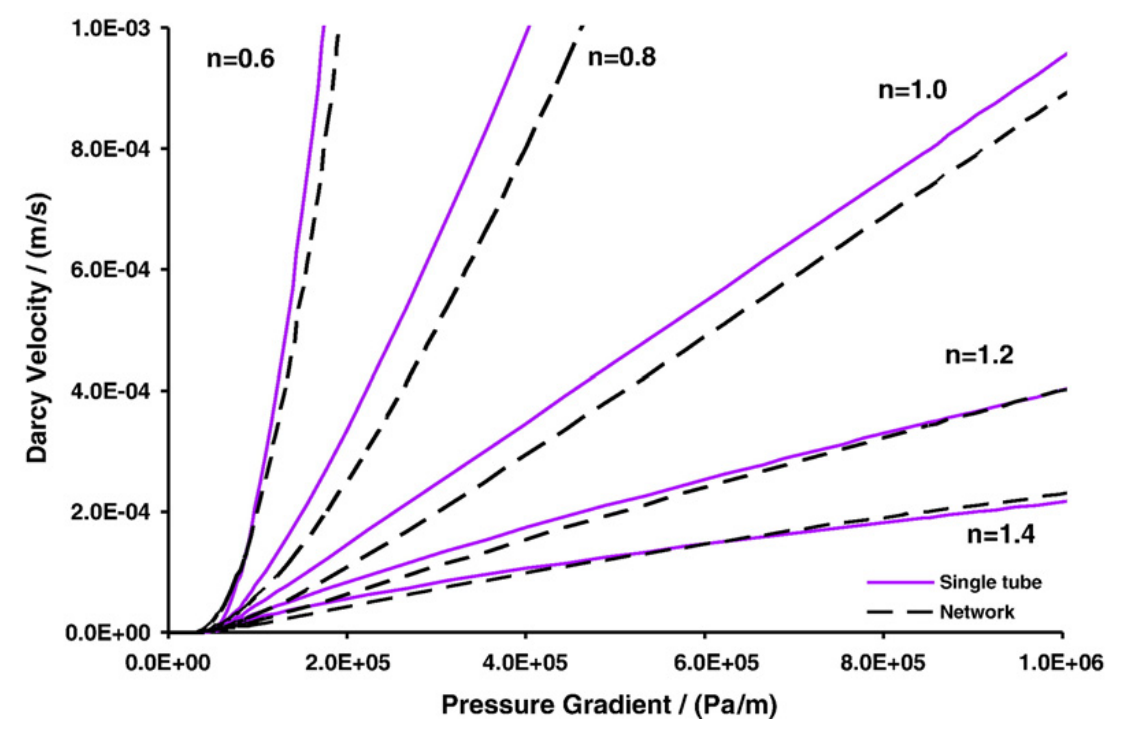

Fig. 2. Comparison between the sand pack network $\left(K=1.0 \times 10^{-10} \mathrm{~m}^{2}, \phi=0.35\right)$ and single tube $(R=48.2 \mu \mathrm{m})$ for a fluid with $\tau_{\mathrm{o}}=1.0 \mathrm{~Pa}$ and $C=0.1$ Pa. s ${ }^{n}$.

the flow hindrance caused by the shear-thickening effect. There are two crossovers: lower and upper. The occurrence and relative location of each crossover is determined by the overall effect of the three factors, some of which are competing. The lower one is caused mainly by the partial blocking of the network plus the shift in the average tube radius relation. The upper one is caused by shear thickening effects because the tube is subject to more shear-thickening at high pressure gradients.

\subsubsection{Berea sandstone network}

The physical and statistical details of this network can be found in Appendix B Table 8. This network is more tortuous and less homogeneous than the sand pack.

The comparison between the results of the Berea network simulation and single tube for the case of a fluid with no yield stress is displayed in Fig. 4. By definition, the network and single tube results are identical in the Newtonian case. Here, in contrast to the sand pack results, the network flow rate is higher than the single tube for shear-thinning fluids $(n<1.0)$. Since the Berea network is more tortuous and heterogeneous, it has a correspondingly higher value of $\alpha$. For $n=0.6, \alpha=1.65$ for a single tube as before, while $\alpha=3.02$ for the network. This gives a higher apparent shear rate in the network, lower viscosity and larger flow rate, as seen in

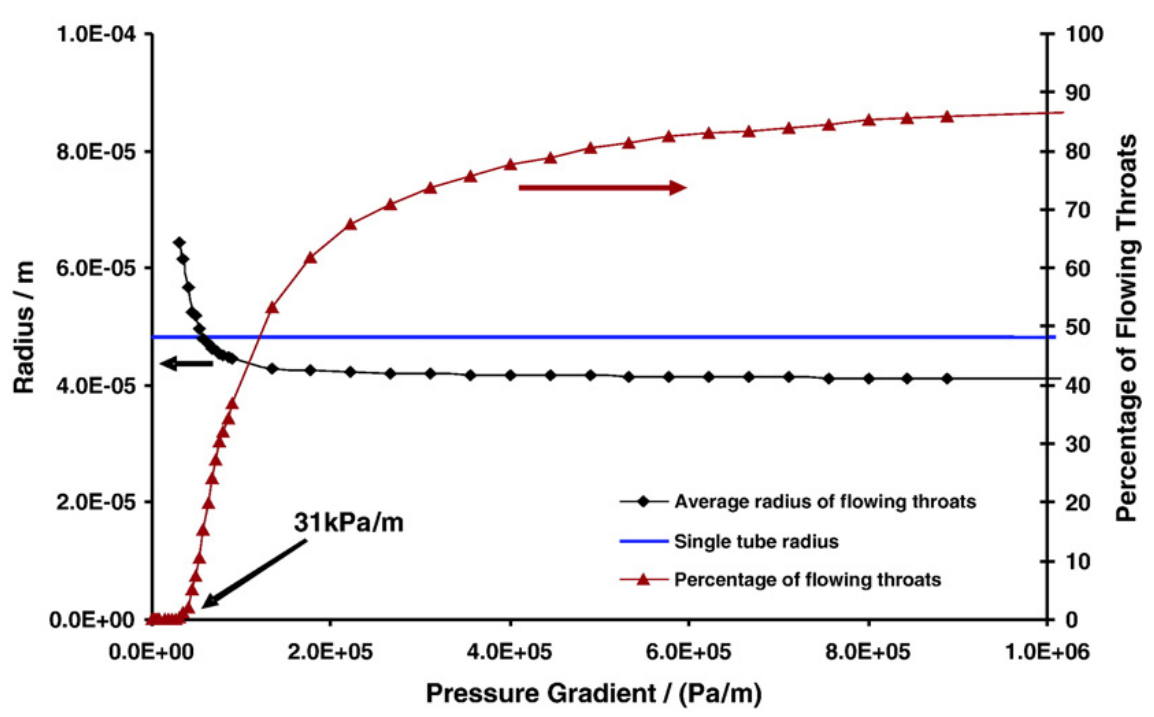

Fig. 3. The radius of the single tube and the average radius of the non-blocked throats of the sand pack network, with their percentage of the total number of throats, as a function of pressure gradient for a Bingham fluid $(n=1.0)$ with $\tau_{\mathrm{o}}=1.0 \mathrm{~Pa}$ and $C=0.1 \mathrm{~Pa}$. s. 


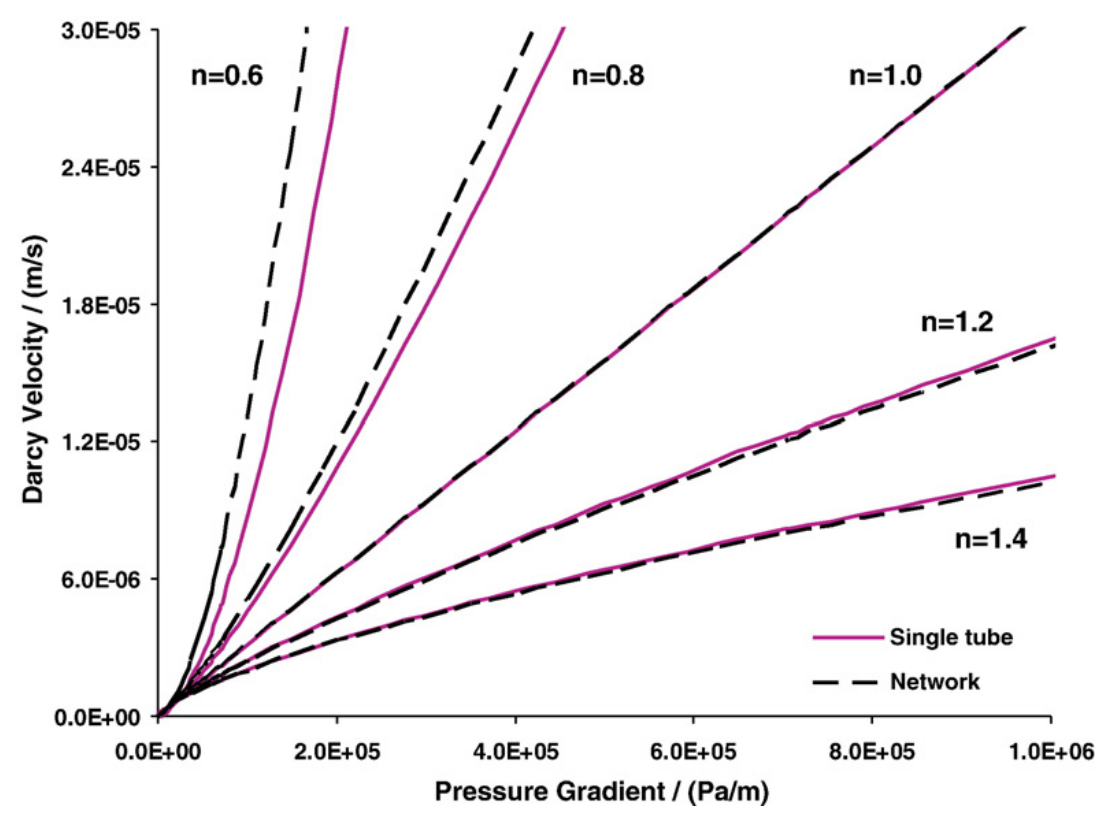

Fig. 4. Comparison between the Berea network $\left(K=3.1 \times 10^{-12} \mathrm{~m}^{2}, \phi=0.19\right)$ and single tube $(R=11.6 \mu \mathrm{m})$ for a fluid with $\tau_{\mathrm{o}}=0.0 \mathrm{~Pa}$ and $C=0.1 \mathrm{~Pa} . \mathrm{s}^{n}$.

Fig. 4. For $n>1.0$, we find, in contrast, that the values of $\alpha$ for the tube and network are similar. For a shearthickening fluid, the viscosity tends to be higher in the throats that carry the largest flow. This tends to make the distribution of flow rates more uniform than for a shearthinning fluid, where flow is enhanced in fast flowing regions. As a consequence the value of $\alpha$ decreases with increasing $\mathrm{n}$ to values similar to that of a single tube.

There is a lack of symmetry between the shear-thinning and shear-thickening cases relative to the Newtonian case, i.e. while the network flow in the shear-thinning cases is considerably higher than the flow in the single tube, the difference between the two flows in the shear-thickening cases is tiny. The reason is the inhomogeneity of the Berea network coupled with the shear effects.

The comparison between the Berea network and the single tube for the case of a fluid with a yield stress is displayed in Fig. 5. As in the sand pack, the Berea network starts flowing before the single tube for the same reason that is the average radius at yield is larger than the single tube radius as seen in Fig. 6.

For a Bingham plastic, the network flow exceeds the single tube flow at low pressure gradients, but the trend is reversed at high pressure gradients for similar reasons as in the sand pack, since some of the elements remain blocked.

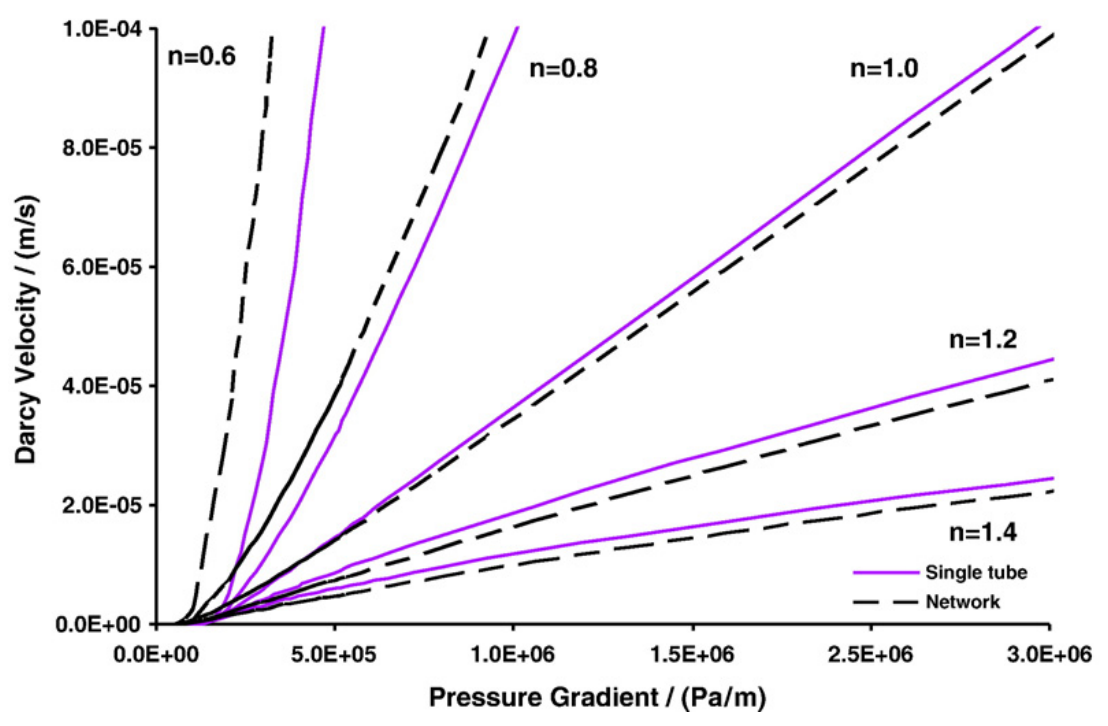

Fig. 5. Comparison between the Berea network $\left(K=3.1 \times 10^{-12} \mathrm{~m}^{2}, \phi=0.19\right)$ and single tube $(R=11.6 \mu \mathrm{m})$ for a Herschel-Bulkley fluid with $\tau_{\mathrm{o}}=1.0 \mathrm{~Pa}$ and $C=0.1 \mathrm{~Pa} . \mathrm{s}^{n}$. 


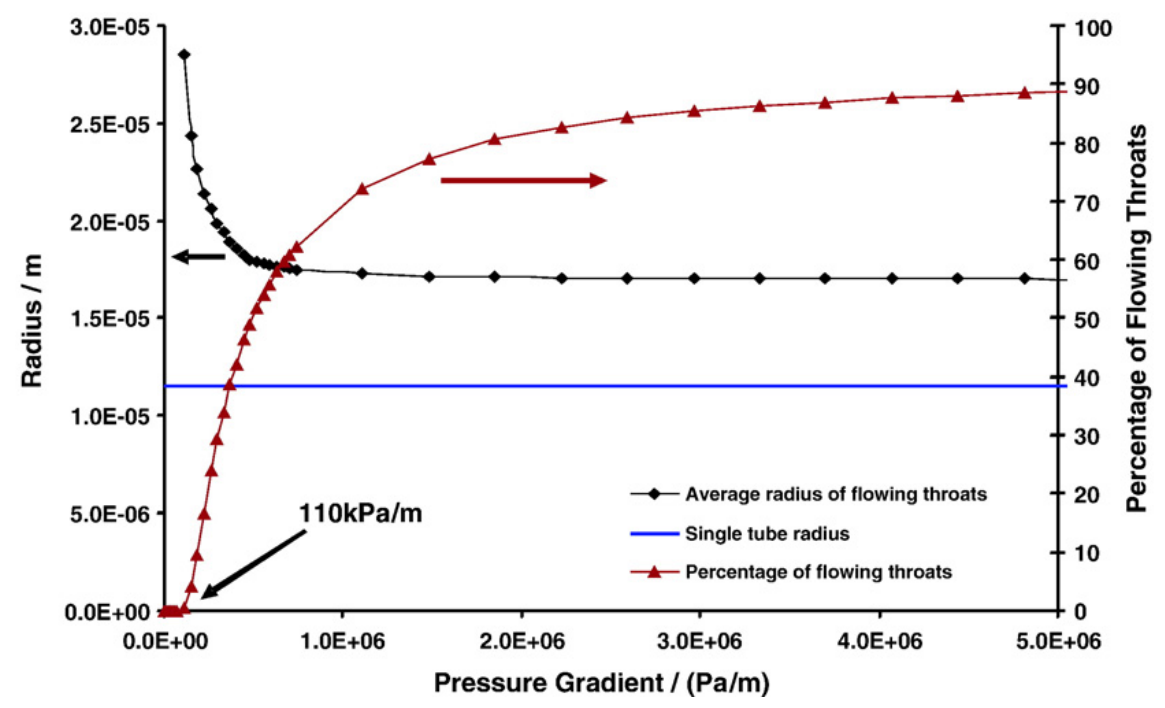

Fig. 6. The radius of the single tube and the average radius of the non-blocked throats of the Berea network, with the percentage of the total number of throats, as a function of pressure gradient for a Bingham fluid $(n=1.0)$ with $\tau_{\mathrm{o}}=1.0 \mathrm{~Pa}$ and $C=0.1 \mathrm{~Pa}$. s.

For the two shear-thinning cases, the general features of the network-single tube relation are similar to those in the case of fluid with no yield stress. However, the discrepancy between the two flows is now larger especially at low pressure gradients. There are three factors affecting the network-single tube relation. The first is the shift because the network yields before the single tube. This factor dominates at low pressure gradients. The second is the inhomogeneity coupled with shear-thinning. The third is the blocking of some network elements with the effect of reducing the total flow. The second and third factors compete, especially at high pressure gradients, and shape the network-single tube relation which can take any form depending on the network and fluid properties and pressure gradient. In our case, the graphs reveal that for the fluid with $n=0.6$ the second factor dominates, while for the fluid with $n=0.8$ the two factors have almost similar impact.

For the two shear-thickening cases, the network flow exceeds the single tube flow at the beginning as the

Table 1

The bulk rheology and bed properties of Sadowski's experimental data

\begin{tabular}{|c|c|c|c|c|c|c|}
\hline \multirow[t]{2}{*}{ Set } & \multicolumn{4}{|l|}{ Fluid properties } & \multicolumn{2}{|c|}{ Bed properties } \\
\hline & Solution & $\mu_{0}$ (Pa. s) & $\alpha$ & $\tau_{1 / 2}(\mathrm{~Pa})$ & $K\left(\mathrm{~m}^{2}\right)$ & $\phi$ \\
\hline 1 & 18.5\% Carbowax 20-M & 0.0823 & 1.674 & 3216.0 & $3.80 \mathrm{E}-09$ & 0.3690 \\
\hline 2 & 18.5\% Carbowax 20-M & 0.0823 & 1.674 & 3216.0 & $1.39 \mathrm{E}-09$ & 0.3812 \\
\hline 3 & $14.0 \%$ Carbowax 20-M & 0.0367 & 1.668 & 3741.0 & $1.38 \mathrm{E}-09$ & 0.3807 \\
\hline 4 & $6.0 \%$ Elvanol $72-51$ & 0.1850 & 2.400 & 1025.0 & 2.63E-09 & 0.3833 \\
\hline 5 & $6.0 \%$ Elvanol $72-51$ & 0.1850 & 2.400 & 1025.0 & $1.02 \mathrm{E}-09$ & 0.3816 \\
\hline 6 & $6.0 \%$ Elvanol $72-51$ & 0.1850 & 2.400 & 1025.0 & 3.93E-09 & 0.3720 \\
\hline 7 & $3.9 \%$ Elvanol $72-51$ & 0.0369 & 1.820 & 2764.0 & $9.96 \mathrm{E}-10$ & 0.3795 \\
\hline 8 & $1.4 \%$ Natrosol-250G & 0.0688 & 1.917 & 59.9 & $2.48 \mathrm{E}-09$ & 0.3780 \\
\hline 9 & $1.4 \%$ Natrosol-250G & 0.0688 & 1.917 & 59.9 & $1.01 \mathrm{E}-09$ & 0.3808 \\
\hline 10 & $1.4 \%$ Natrosol-250G & 0.0688 & 1.917 & 59.9 & 4.17E-09 & 0.3774 \\
\hline 11 & $1.6 \%$ Natrosol-250G & 0.1064 & 1.971 & 59.1 & $2.57 \mathrm{E}-09$ & 0.3814 \\
\hline 12 & $1.6 \%$ Natrosol-250G & 0.1064 & 1.971 & 59.1 & $1.01 \mathrm{E}-09$ & 0.3806 \\
\hline 13 & $1.85 \%$ Natrosol-250G & 0.1670 & 2.006 & 60.5 & $3.91 \mathrm{E}-09$ & 0.3717 \\
\hline 14 & $1.85 \%$ Natrosol-250G & 0.1670 & 2.006 & 60.5 & $1.02 \mathrm{E}-09$ & 0.3818 \\
\hline 15 & $0.4 \%$ Natrosol- $250 \mathrm{H}$ & 0.1000 & 1.811 & 2.2 & $1.02 \mathrm{E}-09$ & 0.3818 \\
\hline 16 & $0.4 \%$ Natrosol- $250 \mathrm{H}$ & 0.1000 & 1.811 & 2.2 & 4.21E-09 & 0.3783 \\
\hline 17 & $0.5 \%$ Natrosol- $250 \mathrm{H}$ & 0.2500 & 2.055 & 3.5 & $1.03 \mathrm{E}-09$ & 0.3824 \\
\hline 18 & $0.5 \%$ Natrosol- $250 \mathrm{H}$ & 0.2500 & 2.055 & 3.5 & $5.30 \mathrm{E}-09$ & 0.3653 \\
\hline 19 & $0.6 \%$ Natrosol- $250 \mathrm{H}$ & 0.4000 & 2.168 & 5.2 & $1.07 \mathrm{E}-09$ & 0.3862 \\
\hline 20 & $0.6 \%$ Natrosol- $250 \mathrm{H}$ & 0.4000 & 2.168 & 5.2 & 5.91E-09 & 0.3750 \\
\hline
\end{tabular}




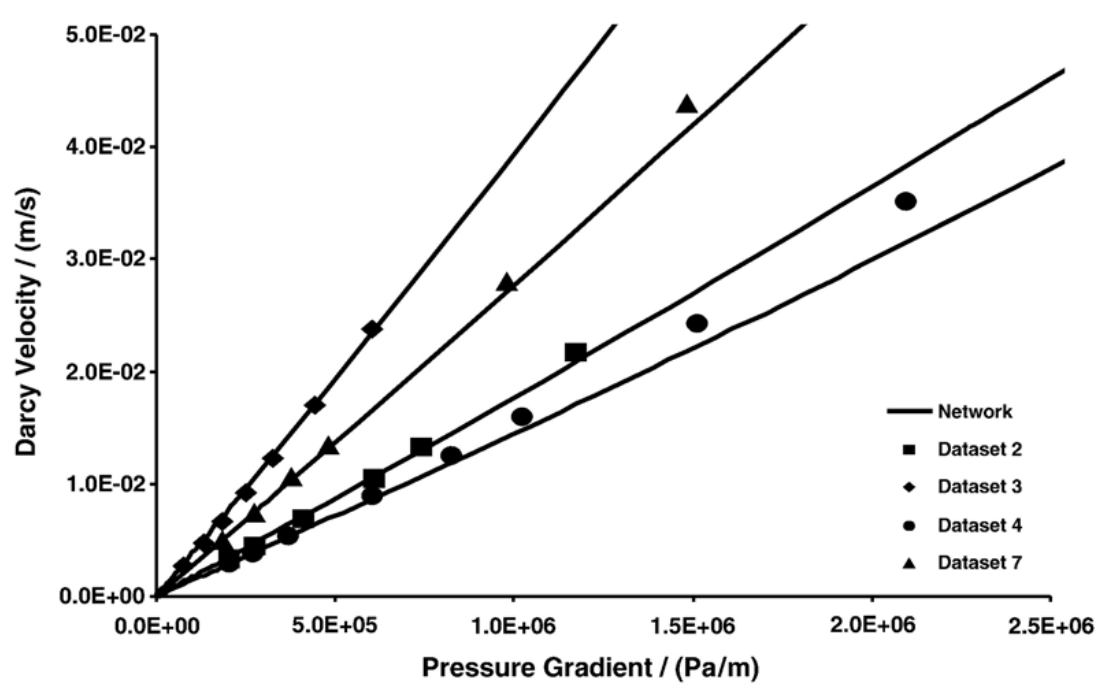

Fig. 7. Sample of the Sadowski's Ellis experimental data sets for a number of solutions with various concentrations and different bed properties alongside the simulation results obtained with scaled sand pack networks having the same permeability.

network yields first, but this eventually is overturned for the same reason as in the Bingham fluid, enforced by the effect of shear-thickening which impedes the flow and gives less flow in the network at high pressure gradients. This is true for Berea because the average radius of the non-blocked throats is greater than the radius of the single tube for all pressure gradients, as seen in Fig. 6, with the effect that the fluid in the network will be subject to more shear-thickening than the fluid in the single tube. Unlike the shear-thinning cases, the inhomogeneity coupled with shear effects and partial blocking of the network are now enforcing each other to deter the flow. The result is that the discrepancy between the network and single tube in the two cases, i.e. $n=1.2$ and $n=1.4$, is larger than that in the corresponding cases of the fluid with no yield stress.

\section{Experimental validation}

In this section, we will discuss the validation of our model by the few complete experimental data collections found in the literature. In all cases presented in this paper, the sand pack network was used after scaling to match the permeability of the porous media of the experiments (Lopez et al., 2003). The reason for using the sand pack instead of Berea is that it is a better, though not ideal, match to the packed beds used in the experiments in terms of homogeneity and tortuosity.

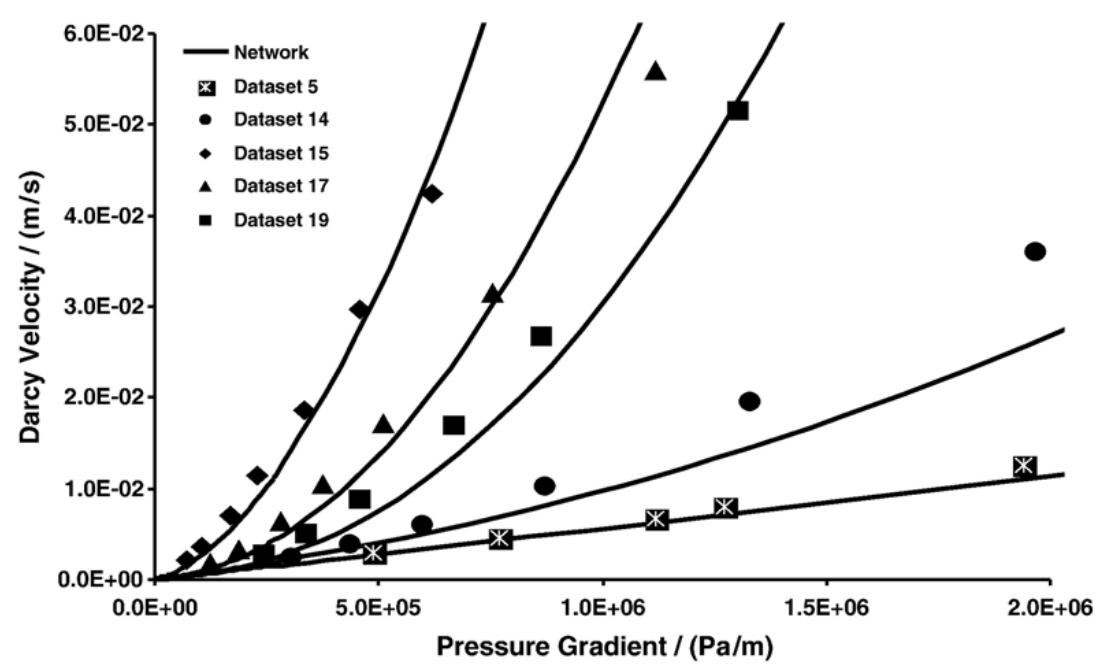

Fig. 8. Sample of the Sadowski's Ellis experimental data sets for a number of solutions with various concentrations and different bed properties alongside the simulation results obtained with scaled sand pack networks having the same permeability. 
Table 2

The bulk rheology of Park's Ellis experimental data

\begin{tabular}{llll}
\hline Solution & $\mu_{0}($ Pa. s) & $\alpha$ & $\tau_{1 / 2}(\mathrm{~Pa})$ \\
\hline $0.50 \%$ & 4.35213 & 2.4712 & 0.7185 \\
$0.25 \%$ & 1.87862 & 2.4367 & 0.5310 \\
$0.10 \%$ & 0.60870 & 2.3481 & 0.3920 \\
$0.05 \%$ & 0.26026 & 2.1902 & 0.3390 \\
\hline
\end{tabular}

\subsection{Ellis model}

Three complete collections of experimental data found in the literature on Ellis fluid were investigated. Good agreement with the network model results was obtained in all cases with no adjustable parameters.

\subsubsection{Sadowski}

In this collection (Sadowski, 1963), twenty complete data sets for ten aqueous polymeric solutions flowing through packed beds of lead shot or glass beads with various properties were investigated. The bulk rheology was given by Sadowski in his dissertation and is shown in Table 1 with the corresponding bed properties. The in-situ experimental data was obtained from the relevant tables in the dissertation. The permeability of the beds, which is needed to scale our sand pack network, was obtained from the formula suggested by Sadowski, that is:

$K=\frac{D_{\mathrm{p}}^{2}}{C} \frac{\varepsilon^{3}}{(1-\varepsilon)^{2}}$

where $K$ is the absolute permeability of the bed, $D_{\mathrm{p}}$ is the diameter of the bed particles, $\varepsilon$ is the porosity and $C$ is a dimensionless constant assigned a value of 180 by Sadowski.

A sample of the simulation results, presented as a Darcy velocity versus pressure gradient, with the corresponding experimental data sets is shown in Figs. 7 and 8 . As seen, the agreement between the experimental data and network simulation is very good in most cases.

\subsubsection{Park}

In this collection (Park, 1972), four complete data sets for the flow of aqueous polyacrylamide solutions with different weight concentration in packed beds of glass beads were investigated. The bulk rheology was given by Park and is shown in Table 2. The in-situ experimental data was obtained from the relevant tables in his dissertation. The permeability of the bed, which is needed to scale our sand pack network, was obtained from Eq. (12), as suggested by Park, with the constant $C$ obtained from fitting his Newtonian flow data. The simulation results compared to the experimental data points are shown in Fig. 9 as a Darcy velocity against pressure gradient. As seen, the agreement in all cases is excellent. The discrepancy observed in some cases in the high-flow rate region is due apparently to the absence of a high-shear Newtonian plateau in the Ellis model.

\subsubsection{Balhoff}

A complete data set for guar gum solution of $0.72 \%$ concentration with Ellis parameters $\mu_{0}=2.672 \mathrm{~Pa}$. s, $\alpha=$ 3.46 and $\tau_{1 / 2}=9.01 \mathrm{~Pa}$ flowing through a packed bed of glass beads having $K=4.19 \times 10^{-9} \mathrm{~m}^{2}$ and $\phi=0.38$ was investigated. The bulk rheology was given by Balhoff

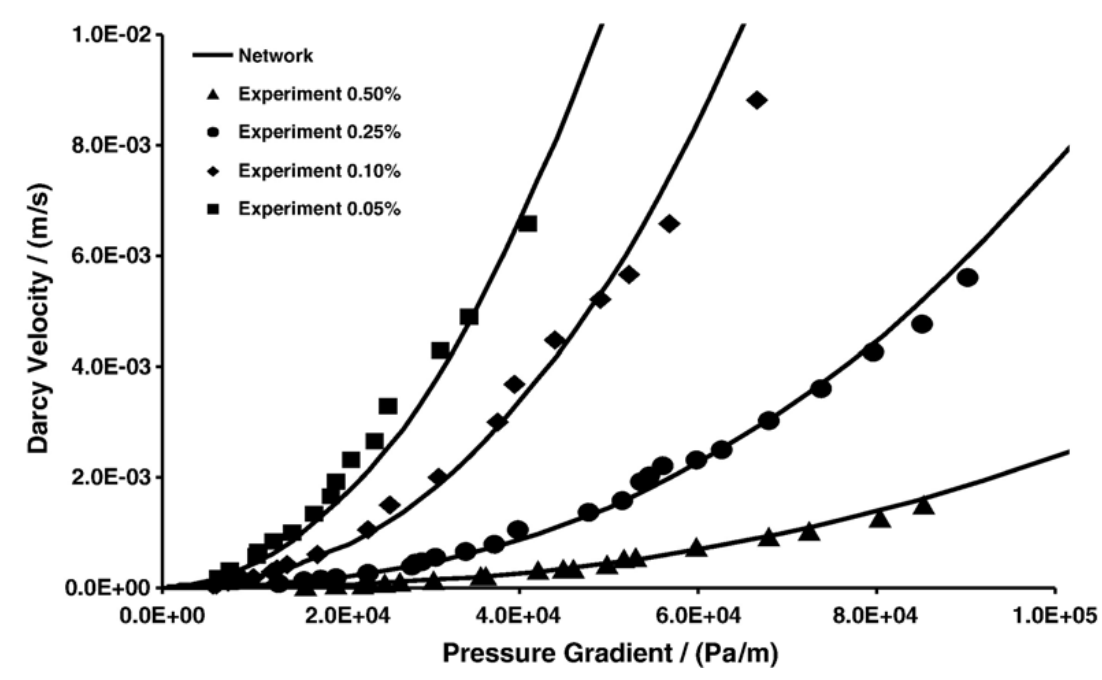

Fig. 9. Park's Ellis experimental data sets for polyacrylamide solutions with 0.50 wt. $\%, 0.25$ wt. $\%, 0.10$ wt. $\%$ and 0.05 wt. $\%$ concentration flowing through a coarse packed bed of glass beads having $K=3.4 \times 10^{-9} \mathrm{~m}^{2}$ and $\phi=0.42$ alongside the simulation results obtained with a scaled sand pack network having the same permeability. 
in his dissertation (Balhoff, 2005) and the in-situ experimental data was obtained from him by private communication.

The simulation results with the experimental data points are shown in Fig. 10 as Darcy velocity versus pressure gradient. As seen, the agreement is very good. Again, a slight discrepancy is observed in the high-flow rate region that may be due to the absence of a highshear Newtonian plateau in the Ellis model.

\subsection{Herschel-Bulkley model}

Three complete collections of experimental data found in the literature on fluid were investigated.

\subsubsection{Park}

In this collection (Park, 1972), eight complete data sets are presented. The fluid is an aqueous solution of Polymethylcellulose (PMC) with two different molecular weights, PMC 25 and PMC 400, each with concentration of $0.3 \mathrm{wt} . \%$ and $0.5 \mathrm{wt} . \%$. For each of the four solutions, two packed beds of spherical uniform-in-size glass beads, coarse and fine, were used.

The in-situ experimental data, alongside the fluids' bulk rheology and the properties of the porous media, were tabulated in Park's thesis. However, the permeability of the two beds, which is needed to scale our network, is missing. To overcome this difficulty, Darcy's law was applied to the Newtonian flow results of the fine bed, as presented in Table M-1 in (Park, 1972), to extract the permeability of this bed. To find the permeability of the coarse bed, Eq. (12) was used with a value of $C$ obtained from the Newtonian flow data.
Table 3

The bulk rheology of Park's Herschel-Bulkley experimental data

\begin{tabular}{llll}
\hline Solution & $C\left(\mathrm{~Pa} . \mathrm{s}^{n}\right)$ & $n$ & $\tau_{\mathrm{o}}(\mathrm{Pa})$ \\
\hline $0.50 \%$ PMC 400 & 0.116 & 0.57 & 0.535 \\
$0.30 \%$ PMC 400 & 0.059 & 0.61 & 0.250 \\
$0.50 \%$ PMC 25 & 0.021 & 0.63 & 0.072 \\
$0.30 \%$ PMC 25 & 0.009 & 0.66 & 0.018 \\
\hline
\end{tabular}

We used our non-Newtonian code with two scaled sand pack networks and the bulk rheology presented in Table 3 to simulate the flow. The simulation results with the corresponding experimental data sets are presented in Figs. 11 and 12. As seen, the predictions are poor. One possible reason is the high-shear-thinning nature of the solutions, with $n$ between 0.57 and 0.66 . This can produce a large discrepancy even for a small error in $n$. The failure to predict the threshold yield pressure is also noticeable. Retention and other similar phenomena may be ruled out as a possible cause for the higher experimental threshold yield pressure by the fact that the solutions, according to Park, were filtered to avoid gel formation.

\subsubsection{Al-Fariss and Pinder}

In this collection (Al-Fariss and Pinder, 1984), there are sixteen complete sets of data for waxy oils with the bulk and in-situ rheologies. The porous media consist of two packed beds of sand having different dimensions, porosity, permeability and grain size. We used the bulk rheology given by the authors and extracted the in-situ rheology from digitized images of the relevant graphs. The bulk rheology and bed properties are given in Table 4.

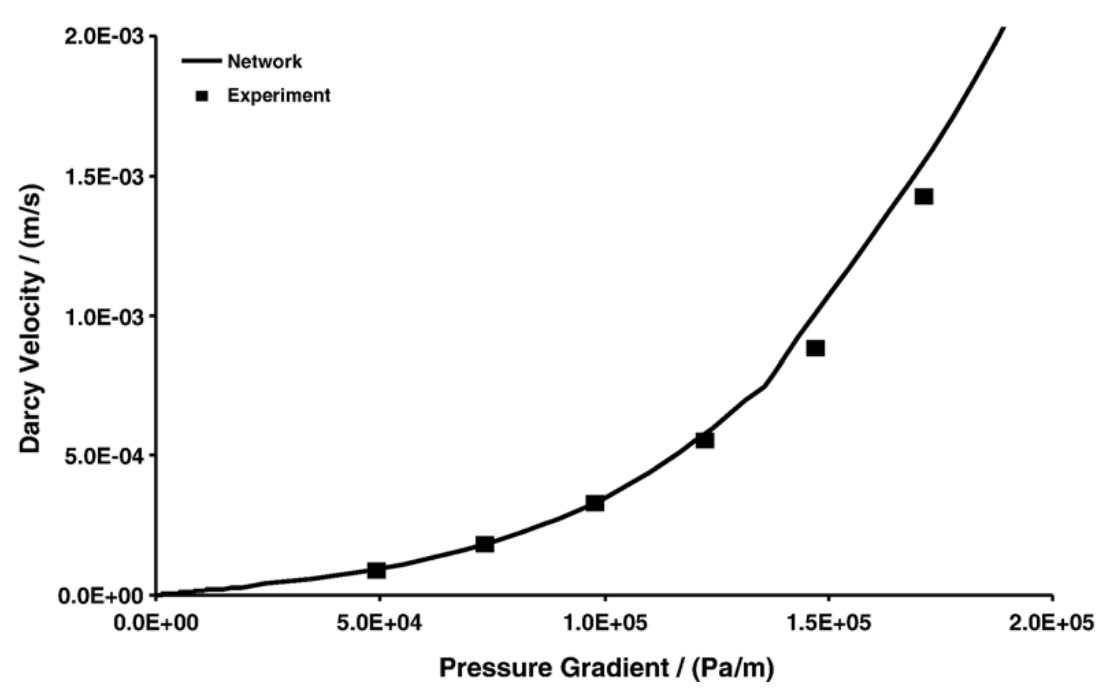

Fig. 10. Balhoff's Ellis experimental data set for guar gum solution with $0.72 \%$ concentration flowing through a packed bed of glass beads having $K=4.2 \times 10^{-9} \mathrm{~m}^{2}$ and $\phi=0.38$ alongside the simulation results obtained with a scaled sand pack network having the same permeability. 


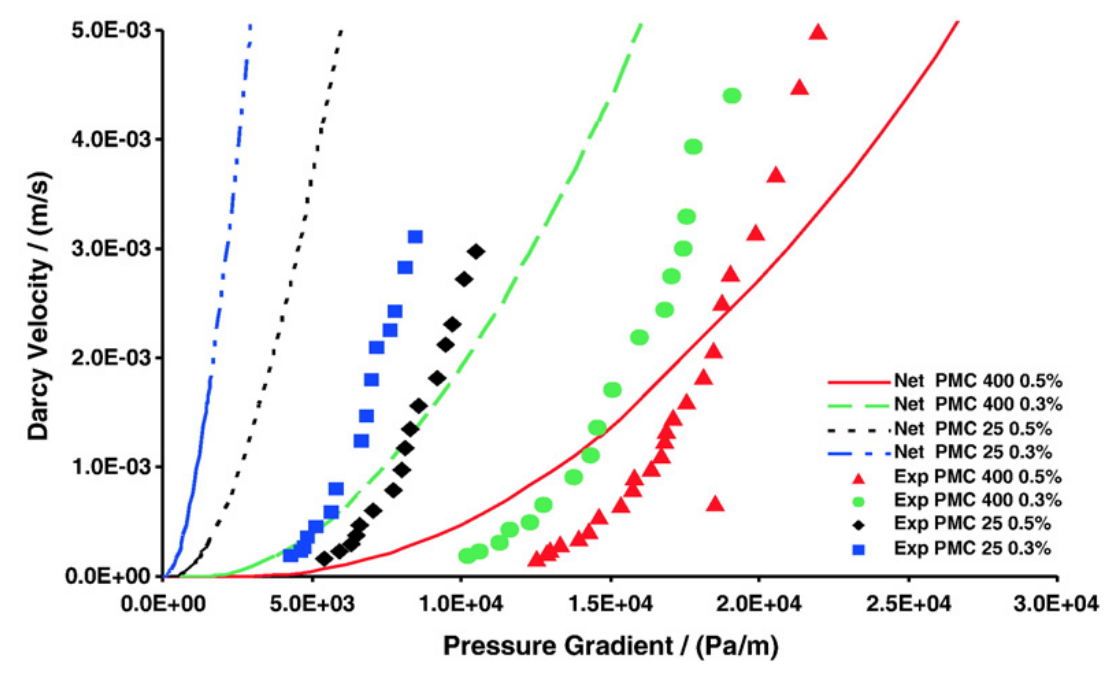

Fig. 11. Park's Herschel-Bulkley experimental data group for aqueous solutions of PMC 400 and PMC 25 with 0.5 wt. $\%$ and 0.3 wt. $\%$ concentration flowing through a coarse packed bed of glass beads having $K=3.4 \times 10^{-9} \mathrm{~m}^{2}$ and $\phi=0.42$ alongside the simulation results obtained with a scaled sand pack network having same permeability.

A sample of the simulation results, with the corresponding in-situ experimental data points, are shown in Figs. 13 and 14. As seen, the agreement in general is better than for the Park data, but still unsatisfactory in places. Analyzing the experimental and network results reveals that while the network behavior is consistent, considering the underlying bulk rheology, the experimental data exhibits an inconsistent pattern. This is evident when looking at the in-situ behavior as a function of the bulk rheology which, in turn, is a function of temperature.

The sixteen data sets are divided into four groups. In each group the fluid and the porous medium are the same but the fluid temperature is different. Analyzing the in-situ data reveals that there is no obvious correlation between the fluid properties and its temperature. An example is the $4.0 \%$ wax in Clarus B group where an increase in temperature from $14{ }^{\circ} \mathrm{C}$ to $16{ }^{\circ} \mathrm{C}$ results in a drop in the flow at high pressures rather than rise, opposite to what is expected from the general trend of the experimental data and the fact that the viscosity usually decreases on increasing the temperature. One possibility is that in some cases the wax-oil mix may not be homogenous, so other physical phenomena, such as wax precipitation, took place. Such complex phenomena are not accounted for in our model.

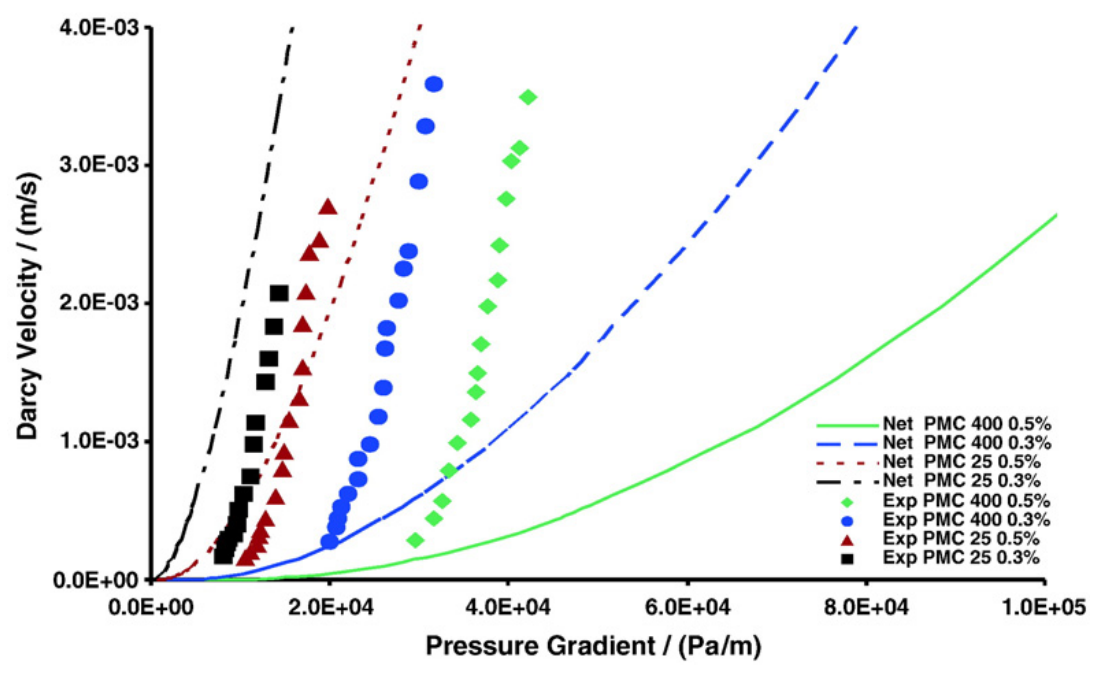

Fig. 12. Park's experimental data group for aqueous solutions of PMC 400 and PMC 25 with 0.5 wt. $\%$ and 0.3 wt. $\%$ concentration flowing through a fine packed bed of glass beads having $K=3.6 \times 10^{-10} \mathrm{~m}^{2}$ and $\phi=0.39$ alongside the simulation results obtained with a scaled sand pack network having the same permeability. 
Table 4

The bulk rheology and bed properties of the experimental data of AlFariss and Pinder

\begin{tabular}{lllllllll}
\hline \multicolumn{2}{l}{ Fluid properties } & & & & & \multicolumn{2}{l}{ Bed properties } \\
\cline { 1 - 1 } \cline { 8 - 9 } Wax $(\%)$ & $T\left({ }^{\circ} \mathrm{C}\right)$ & $C\left(\mathrm{~Pa} . \mathrm{s}^{n}\right)$ & $n$ & $\tau_{\mathrm{o}}(\mathrm{Pa})$ & & $K\left(\mathrm{~m}^{2}\right)$ & $\phi$ \\
\hline 2.5 & 10 & 0.675 & 0.89 & 0.605 & & $3.15 \mathrm{E}-10$ & 0.36 \\
2.5 & 12 & 0.383 & 0.96 & 0.231 & & $3.15 \mathrm{E}-10$ & 0.36 \\
2.5 & 14 & 0.300 & 0.96 & 0.142 & & $3.15 \mathrm{E}-10$ & 0.36 \\
2.5 & 18 & 0.201 & 0.97 & 0.071 & & $3.15 \mathrm{E}-10$ & 0.36 \\
4.0 & 12 & 1.222 & 0.77 & 3.362 & & $3.15 \mathrm{E}-10$ & 0.36 \\
4.0 & 14 & 0.335 & 0.97 & 3.150 & & $3.15 \mathrm{E}-10$ & 0.36 \\
4.0 & 16 & 0.461 & 0.88 & 1.636 & & $3.15 \mathrm{E}-10$ & 0.36 \\
4.0 & 18 & 0.436 & 0.85 & 0.480 & & $3.15 \mathrm{E}-10$ & 0.36 \\
4.0 & 20 & 0.285 & 0.90 & 0.196 & & $3.15 \mathrm{E}-10$ & 0.36 \\
5.0 & 16 & 0.463 & 0.87 & 3.575 & & $3.15 \mathrm{E}-10$ & 0.36 \\
5.0 & 18 & 0.568 & 0.80 & 2.650 & & $3.15 \mathrm{E}-10$ & 0.36 \\
5.0 & 20 & 0.302 & 0.90 & 1.921 & & $3.15 \mathrm{E}-10$ & 0.36 \\
Crude & 2 & 0.673 & 0.54 & 2.106 & & $1.58 \mathrm{E}-09$ & 0.44 \\
Crude & 8 & 0.278 & 0.61 & 0.943 & & $1.58 \mathrm{E}-09$ & 0.44 \\
Crude & 10 & 0.127 & 0.70 & 0.676 & & $1.58 \mathrm{E}-09$ & 0.44 \\
Crude & 14 & 0.041 & 0.81 & 0.356 & & $1.58 \mathrm{E}-09$ & 0.44 \\
\hline
\end{tabular}

\subsubsection{Chase and Dachavijit}

In this collection (Chase and Dachavijit, 2003), there are ten complete data sets for Bingham aqueous solutions of Carbopol 941 with concentration varying between 0.15 and $1.3 \%$. The porous medium is a packed column of spherical glass beads having a narrow size distribution. The bulk rheology, which is extracted from a digitized image and given in Table 5, represents the actual experimental data points rather than the least square fitting suggested by the authors.

Our non-Newtonian code was used to simulate the flow of a Bingham fluid with the extracted bulk rheology through a scaled sand pack network. The scaling factor was chosen to have a permeability that produces a best fit to the most Newtonian-like data set, excluding the first data set with the lowest concentration due to a very large relative error and a lack of fit to the trend line.

The simulation results, with the corresponding in-situ experimental data sets extracted from digitized images of the relevant graphs, are presented in Figs. 15 and 16. The fit is good in most cases. The experimental data in some cases shows irregularities which may suggest large experimental errors or other physical phenomena taking place such as retention. This erratic behavior cannot fit a consistent pattern.

\section{Yield-stress analysis}

For yield-stress fluids, a certain amount of stress must be exceeded before the flow starts (Bear, 1972). So the substance is a solid before yield and a fluid after. There are several difficulties in working with the yield-stress fluids and validating the experimental data. One difficulty is that the yield-stress value is usually obtained by extrapolating a plot of shear stress to zero shear rate (Park et al., 1973; Al-Fariss and Pinder, 1984; Carreau et al., 1997; Balhoff, 2005). This extrapolation can result in a variety of values for yield stress, depending on the distance from the shear stress axis experimentally accessible by the instrument used. The vast majority of yieldstress data reported results from such extrapolations, making most values in the literature instrument-dependent (Carreau et al., 1997).

A shortcoming of using continuum models, such as an extended Darcy's law, to study the flow of yield-stress fluids in porous media is that these models are unable to

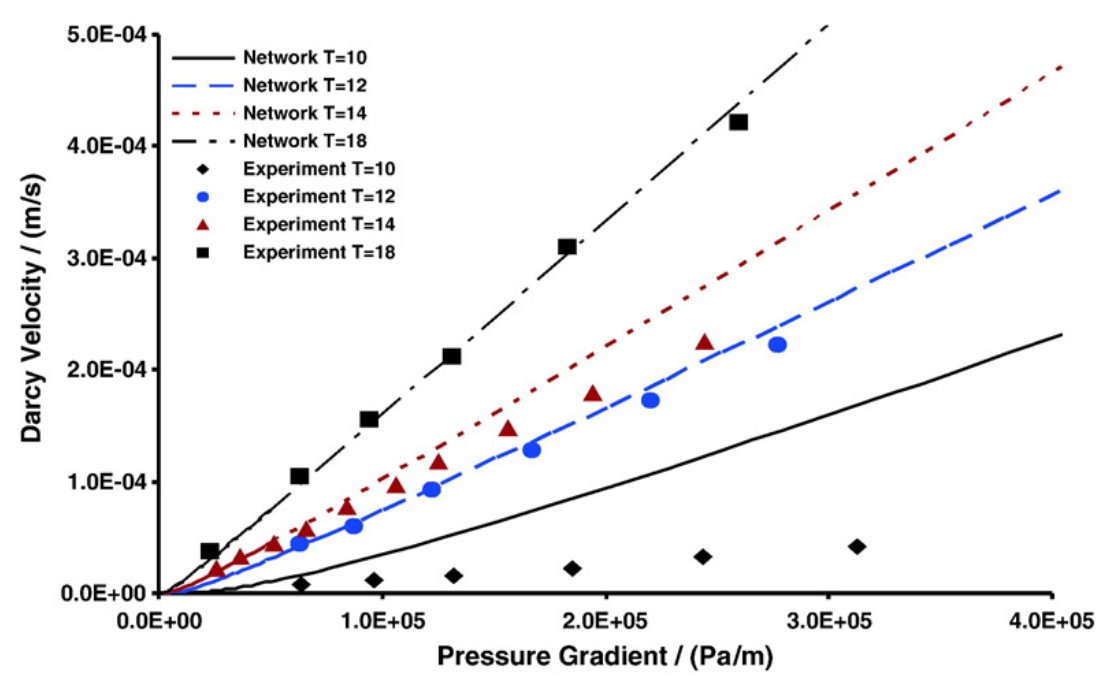

Fig. 13. Al-Fariss and Pinder's Herschel-Bulkley experimental data group for $2.5 \%$ wax in Clarus B oil flowing through a column of sand having $K=3.1 \times 10^{-10} \mathrm{~m}^{2}$ and $\phi=0.36$ alongside the simulation results obtained with a scaled sand pack network having the same $K$ and $\phi$. The temperatures, $T$, are in ${ }^{\circ} \mathrm{C}$. 


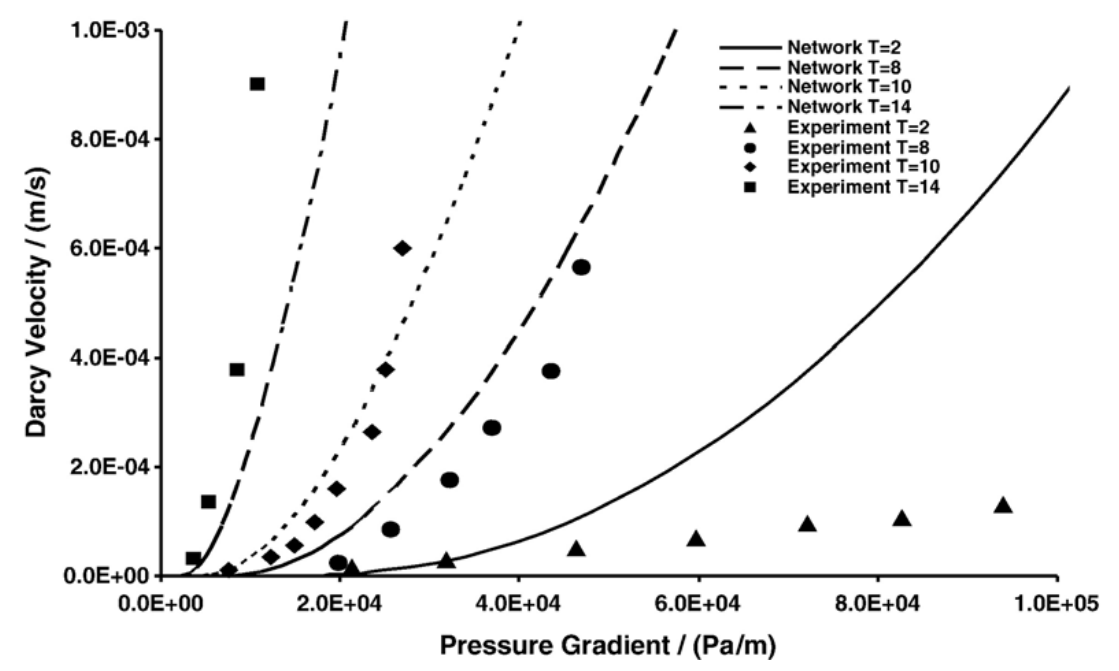

Fig. 14. Al-Fariss and Pinder's Herschel-Bulkley experimental data group for waxy crude oil flowing through a column of sand having $K=1.6 \times 10^{-9} \mathrm{~m}^{2}$ and $\phi=0.44$ alongside the simulation results obtained with a scaled sand pack network having the same permeability. The temperatures, $T$, are in ${ }^{\circ} \mathrm{C}$.

correctly describe the network behavior at transition where the network is partly flowing, because according to these models the network is either fully blocked or fully flowing whereas in reality the network smoothly yields. For instance, these models predict for Bingham fluids a linear relationship between Darcy velocity and pressure gradient with an intercept at threshold yield gradient whereas our network model and that of others (Balhoff and Thompson, 2004), supported by experimental evidence, predict a nonlinear behavior at transition stage (Vradis and Protopapas, 1993), see Figs. 2 and 5. Some authors have concluded that in a certain range the macroscopic flow rate of Bingham plastic in a network depends quadratically on the departure of the applied pressure difference from its minimum value (Chen et al., 2005b), which is broadly consistent with our network predictions.

\subsection{Predicting the yield pressure of the network}

Predicting the threshold yield pressure of a yield-stress fluid in porous media is a special case of the more general problem of finding the threshold conduction path in disordered media consisting of elements with randomly distributed thresholds. This problem was analyzed by Kharabaf and Yortsos (1996), and Roux and Hansen (1987) in the context of studying the conduction of an electric network of diodes by considering two different cases, one in which the path is directed (no backtracking) and one in which it is not. They suggested that the minimum overall threshold potential difference across the network is akin to a percolation threshold and studied its dependence on the lattice size.

Kharabaf and Yortsos (1996) noticed that a firm connection of the lattice-threshold problem to percola- tion appears to be lacking and the relation of the Minimum Threshold Path (MTP) to the minimum path of percolation, if it indeed exists, is not self-evident. They presented a new algorithm, Invasion Percolation with Memory (IPM), for the construction of the MTP, based on which its properties can be studied. This algorithm will be examined later on.

In a series of studies on generation and mobilization of foam in porous media, Rossen and Gauglitz (1990), Rossen and Mamun (1993) analyzed the threshold yield pressure using percolation theory concepts and suggested a simple percolation model. In this model, the percolation cluster is first found, then the MTP was approximated as a subset of this cluster that samples those bonds with the smallest individual thresholds (Chen et al., 2005b).

Chen et al. (2005b) extended the Invasion Percolation with Memory method of Kharabaf and Yortsos to incorporate dynamic effects due to the viscous friction following the onset of mobilization.

Table 5

The bulk rheology of Chase and Dachavijit experimental data for a Bingham fluid $(n=1.0)$

\begin{tabular}{llc}
\hline Concentration (\%) & $C$ (Pa. s) & $\tau_{\mathrm{o}}(\mathrm{Pa})$ \\
\hline 0.15 & 0.003 & 0.08 \\
0.37 & 0.017 & 2.06 \\
0.40 & 0.027 & 2.39 \\
0.45 & 0.038 & 4.41 \\
0.54 & 0.066 & 4.37 \\
0.60 & 0.057 & 7.09 \\
0.65 & 0.108 & 8.70 \\
0.86 & 0.136 & 12.67 \\
1.00 & 0.128 & 17.33 \\
1.30 & 0.215 & 28.46 \\
\hline
\end{tabular}




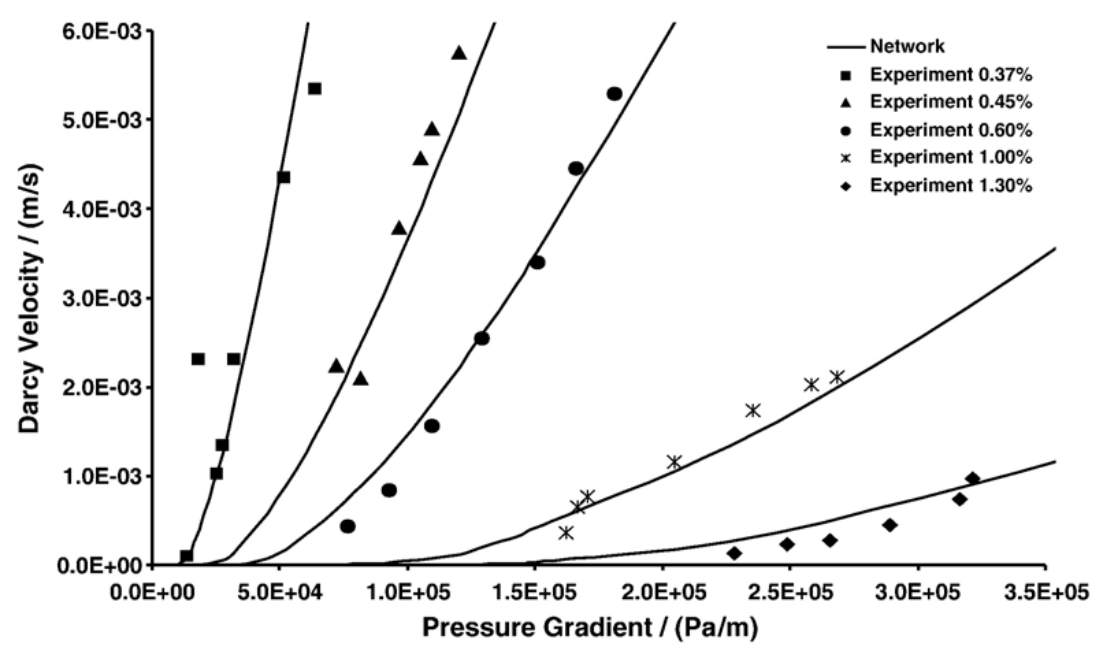

Fig. 15. Network simulation results with the corresponding experimental data points of Chase and Dachavijit for a Bingham aqueous solution of Carbopol 941 with various concentrations $(0.37 \%, 0.45 \%, 0.60 \%, 1.00 \%$ and $1.30 \%)$ flowing in a packed column of glass beads.

In this paper, two approaches to predict the network threshold yield pressure are presented: the Invasion Percolation with Memory of Kharabaf and Yortsos (1996), and the Path of Minimum Pressure which is a novel approximate approach that we suggest. Both approaches are implemented in our three-dimensional network model code. An analysis is conducted to compare the prediction and performance of these approaches and relate their results to the network threshold yield pressure as obtained from flow simulation.

\subsubsection{Invasion Percolation with Memory (IPM)}

This method is a way of finding the inlet-to-outlet path that minimizes the sum of the values of a property assigned to the individual elements of the network, and hence finding this minimum. For a yield-stress fluid, this reduces to finding the inlet-to-outlet path that minimizes the yield pressure. The yield pressure of this path is taken as the network threshold yield pressure. An algorithm to find the threshold yield pressure according to IPM is outlined below:

1. Initially, the nodes on the inlet are considered to be sources and the nodes on the outlet and inside are targets. The inlet nodes are assigned a pressure value of 0.0. According to the IPM, a source cannot be a target and vice versa, i.e. they are disjoint sets and remain so in all stages.

2. Starting from the source nodes, a step forward is made in which the yield front advances one bond from a single source node. The condition for choosing this step is that the sum of the source pressure plus the yield pressure of the bond connecting the source to the target node is the minimum of all similar sums from

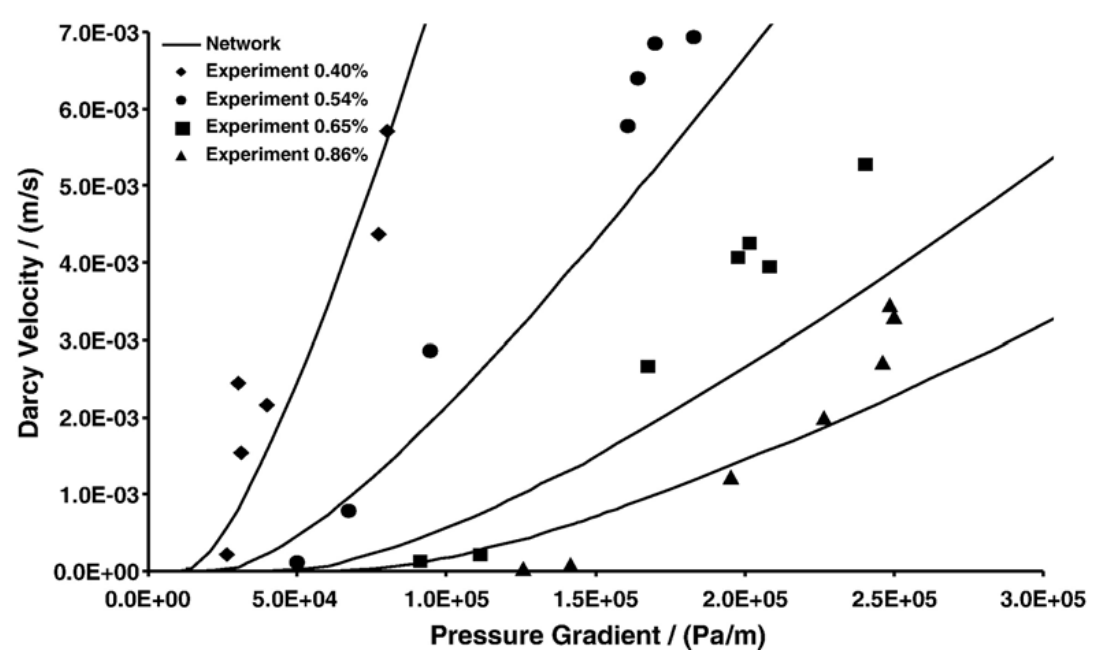

Fig. 16. Network simulation results with the corresponding experimental data points of Chase and Dachavijit for a Bingham aqueous solution of Carbopol 941 with various concentrations $(0.40 \%, 0.54 \%, 0.65 \%$ and $0.86 \%)$ flowing in a packed column of glass beads. 
the source nodes to the possible target nodes. This sum is assigned to the target node.

3. This target node loses its status as a target and obtains the status of a source.

4. The last two steps are repeated until the outlet is reached, i.e. when the target is being an outlet node. The pressure assigned to this target node is regarded as the yield pressure of the network.

We implemented this algorithm in our non-Newtonian code. A sample of the results is presented in Table 6. As implemented, the memory requirement for a network with $N$ nodes is of order $N$ which is a trivial cost even for a large network. The CPU time for a medium-size network however is considerable and scales approximately as $N^{2}$.

\subsubsection{Path of Minimum Pressure (PMP)}

This is a novel approach that we developed. It is based on a similar assumption to that upon which the IPM is based, that is the network threshold yield pressure is the minimum sum of the threshold yield pressures of the individual elements of all possible paths from the inlet to the outlet. However, it is computationally more efficient than the IPM in terms of time and memory.

According to the PMP, to find the threshold yield pressure of a network:

1. All possible paths of serially-connected bonds from inlet to outlet are inspected. We impose a condition on the spanning path that there is no flow component opposite to the pressure gradient across the network in any part of the path, i.e. backtracking is not allowed.

2. For each path, the threshold yield pressure of each bond is computed and the sum of these pressures is found.
3. The network threshold yield pressure is taken as the minimum of these sums.

This algorithm was implemented in our non-Newtonian code and a sample of the results is given in Table 6. The CPU time is trivial even for a relatively large network and rises almost linearly with the size of the network. Typically IPM took around $400 \mathrm{~s}$ to converge, as compared to only $6 \mathrm{~s}$ for PMP.

\subsubsection{Analyzing IPM and PMP}

The two methods are used to investigate the threshold yield pressure of various slices of the sand pack and Berea networks with different location and width. A sample of the results is presented in Table 6.

In many cases the predictions of the IPM and PMP agree. However, when they disagree the PMP gives the higher value of yield pressure. The reason is that backtracking is allowed in IPM but not in PMP. When the actual path of minimum sum has a backward component, which is not allowed by the PMP, the alternative path of next minimum sum with no backtracking is more restrictive and hence has a higher yield pressure value.

The numerically computed onset of flow is similar to the IPM and PMP thresholds for the sand pack, although slightly higher. In the network, we first see flow at a finite flow rate where the flow paths may be multiply connected with some elements with pressure gradients beyond the yield value. For the Berea network, the numerically computed threshold is two to three times higher than predicted. This implies a slow onset of flow, evident in Figs. 2 and 5, where there is a very low flow rate over a wide range of pressure gradient. This implies that the accurate assessment of a yield threshold may be difficult

Table 6

Comparison of the yield pressure, $P_{\mathrm{y}}$, predicted by IPM and PMP compared to the flow simulation value computed for various slices of the sand pack and Berea networks

\begin{tabular}{|c|c|c|c|c|c|c|c|c|}
\hline \multirow[b]{2}{*}{ No. } & \multicolumn{2}{|c|}{ Boundaries } & \multicolumn{3}{|c|}{$P_{\mathrm{y}}(\mathrm{Pa})$ of sand pack } & \multicolumn{3}{|c|}{$P_{\mathrm{y}}(\mathrm{Pa})$ of Berea } \\
\hline & Lower & Upper & Network & IPM & PMP & Network & IPM & PMP \\
\hline 1 & 0.0 & 1.0 & 80.94 & 53.81 & 54.92 & 331.49 & 121.68 & 121.68 \\
\hline 2 & 0.0 & 0.9 & 71.25 & 49.85 & 51.13 & 251.20 & 102.98 & 104.46 \\
\hline 3 & 0.0 & 0.8 & 61.14 & 43.96 & 44.08 & 235.15 & 84.55 & 87.51 \\
\hline 4 & 0.0 & 0.7 & 56.34 & 38.47 & 38.74 & 148.47 & 62.32 & 62.32 \\
\hline 5 & 0.1 & 1.0 & 69.75 & 47.67 & 48.78 & 283.88 & 119.38 & 119.38 \\
\hline 6 & 0.1 & 0.9 & 60.24 & 43.71 & 44.70 & 208.97 & 100.67 & 102.15 \\
\hline 7 & 0.1 & 0.8 & 49.53 & 37.82 & 37.94 & 189.73 & 82.24 & 84.23 \\
\hline 8 & 0.2 & 1.0 & 53.81 & 43.26 & 43.86 & 295.77 & 110.33 & 115.60 \\
\hline 9 & 0.2 & 0.9 & 46.44 & 39.30 & 40.19 & 222.84 & 94.58 & 95.48 \\
\hline 10 & 0.3 & 1.0 & 53.19 & 37.85 & 37.85 & 295.95 & 96.73 & 102.00 \\
\hline
\end{tabular}

The data is for a Bingham fluid $(n=1.0)$ with $\tau_{\mathrm{o}}=1.0 \mathrm{~Pa}$. 
to obtain experimentally and numerically. While it is possible to compute flow at lower pressure gradients numerically by using IPM or PMP to predict which elements first yield, the resultant flow rates are very low.

\section{Discussion and conclusions}

Some results and conclusions that can be drawn from this work are:

- The network model has been extended to account for different types of non-Newtonian rheology: Ellis and Herschel-Bulkley models. The basis of the implementation is an analytical expression for the nonlinear relationship between flow rate and pressure drop in a single cylindrical element. These expressions are combined in an iterative technique to find the total flow across the network.

- The general trends in behavior for shear-independent, shear-thinning and shear-thickening fluids with and without yield stress have been discussed. Compared to an equivalent single tube, our networks yield at a lower pressure gradient. However, some elements remain blocked even at very high pressure gradients. Shearthinning accentuates the heterogeneity of the network, while shear-thickening makes the flow more uniform.

- The network model successfully predicted several experimental data sets reported in the literature on Ellis fluids.

- The model predicted data for yield-stress fluids with mixed results. The discrepancies were likely to be due to physical effects we did not model such as precipitation and adsorption.

- We implemented two methods to predict the network yield pressure: IPM and a new technique PMP that is more computationally efficient with the assumption that there is no backtracking. The two methods gave similar predictions which are close to the numerically computed onset of flow in the sand pack network. For Berea, the numerically computed onset of flow occurred at a higher pressure gradient than predicted by IPM or PMP. This implies that there is a slow onset of flow with very little flow over a wide range of pressure gradient.

$\begin{array}{ll}\text { Nomenclature } \\ \text { Symbol } & \text { Meaning } \\ \alpha & \text { parameter in Ellis model } \\ \alpha & \text { scale factor } \\ \gamma & \text { shear rate } \\ \Delta P & \text { pressure drop } \\ \Delta P_{\text {th }} & \text { threshold pressure drop } \\ \varepsilon & \text { porosity } \\ \mu & \text { viscosity }\end{array}$

\begin{tabular}{|c|c|c|c|}
\hline$\mu_{\text {app }}$ & apparent viscosity & $\mathrm{ML}^{-1} \mathrm{~T}^{-1}$ & Pa. s \\
\hline$\mu_{\text {eff }}$ & effective viscosity & $\mathrm{ML}^{-1} \mathrm{~T}^{-1}$ & Pa. s \\
\hline$\mu_{0}$ & low-shear viscosity & $\mathrm{ML}^{-1} \mathrm{~T}^{-1}$ & Pa. s \\
\hline$\tau$ & shear stress & $\mathrm{ML}^{-1} \mathrm{~T}^{-2}$ & $\mathrm{~Pa}$ \\
\hline$\tau_{1 / 2}$ & shear stress when $\mu=\mu_{0} / 2$ & $\mathrm{ML}^{-1} \mathrm{~T}^{-2}$ & $\mathrm{~Pa}$ \\
\hline$\tau_{\mathrm{o}}$ & yield stress & $\mathrm{ML}^{-1} \mathrm{~T}^{-2}$ & $\mathrm{~Pa}$ \\
\hline$\tau_{\mathrm{w}}$ & stress at tube wall $(=\Delta P R / 2 L)$ & $\mathrm{ML}^{-1} \mathrm{~T}^{-2}$ & $\mathrm{~Pa}$ \\
\hline$\phi$ & porosity & no dimensions & no units \\
\hline$\nabla P$ & pressure gradient & $\mathrm{ML}^{-2} \mathrm{~T}^{-2}$ & Pa. $\mathrm{m}^{-1}$ \\
\hline$C$ & consistency factor & $\mathrm{ML}^{-1} \mathrm{~T}^{n-2}$ & Pa. $s^{n}$ \\
\hline$C$ & packed bed parameter & no dimensions & no units \\
\hline$D_{\mathrm{p}}$ & particle diameter & $\mathrm{L}$ & $\mathrm{m}$ \\
\hline $\mathrm{d} r$ & infinitesimal change in radius & $\mathrm{L}$ & $\mathrm{m}$ \\
\hline$G$ & conductance & $\mathrm{L}^{4}$ & $\mathrm{~m}^{4}$ \\
\hline$K$ & absolute permeability & $\mathrm{L}^{2}$ & $\mathrm{~m}^{2}$ \\
\hline$L$ & tube length & $\mathrm{L}$ & $\mathrm{m}$ \\
\hline$n$ & flow behavior index & no dimensions & no units \\
\hline$P$ & pressure & $\mathrm{ML}^{-1} \mathrm{~T}^{-2}$ & $\mathrm{~Pa}$ \\
\hline$P_{\mathrm{y}}$ & yield pressure & $\mathrm{ML}^{-1} \mathrm{~T}^{-2}$ & $\mathrm{~Pa}$ \\
\hline$q$ & Darcy velocity & $\mathrm{LT}^{-1}$ & $\mathrm{~m} \cdot \mathrm{s}^{-1}$ \\
\hline$Q$ & volumetric flow rate & $\mathrm{L}^{3} \mathrm{~T}^{-1}$ & $\mathrm{~m}^{3} \cdot \mathrm{s}^{-1}$ \\
\hline$r$ & radius & $\mathrm{L}$ & $\mathrm{m}$ \\
\hline$R$ & tube radius & $\mathrm{L}$ & $\mathrm{m}$ \\
\hline$R_{e q}$ & equivalent radius & $\mathrm{L}$ & $\mathrm{m}$ \\
\hline$t$ & time & $\mathrm{T}$ & $\mathrm{s}$ \\
\hline$T$ & temperature & $\Theta$ & $\mathrm{K},{ }^{\circ} \mathrm{C}$ \\
\hline
\end{tabular}

Abbreviations and notations:

app apparent

Ave. Average

eff effective

eq equivalent

Eq. Equation

Exp Experimental

iff if and only if

IPM Invasion Percolation with Memory

m meter

Max. Maximum

Min. Minimum

$\mathrm{mm}$ millimeter

MTP Minimum Threshold Path

Net Network

No. Number

PMP Path of Minimum Pressure

St. Dev. Standard Deviation

th threshold

$\mu \mathrm{m} \quad$ micrometer

| $\quad$ modulus

\section{Acknowledgements}

The authors would like to thank all the members of the Imperial College Consortium on Pore-Scale Modeling (BHP, DTI, ENI, JOGMEC, Saudi Aramco, Schlumberger, Shell, Statoil and Total) for financial support of our research. 


\section{Appendix A. Derivation of flow rate of Ellis and Herschel-Bulkley fluids in cylindrical tube}

Here, we outline derivations of analytical expressions for the volumetric flow rate in a cylindrical duct, with inner radius $R$ and length $L$, using cylindrical polar coordinate system where the tube axis coincides with the coordinate $z$-axis and the flow is in the positive $z$-direction.

We apply the well-known general result, sometimes called the Weissenberg-Rabinowitsch equation, which relates the flow rate $Q$ and the shear stress at the tube wall $\tau_{\mathrm{w}}$ for laminar flow of time-independent fluid. This equation, which can be derived considering the volumetric flow rate through the differential annulus between radii $r$ and $r+\mathrm{d} r$, is (Skelland, 1967; Bird et al., 1987; Carreau et al., 1997):

$\frac{Q}{\pi R^{3}}=\frac{1}{\tau_{\mathrm{w}}^{3}} \int_{0}^{\tau_{\mathrm{w}}} \tau^{2} \gamma \mathrm{d} \tau$

where $\tau$ is the shear stress and $\gamma$ is the shear rate.

For an Ellis fluid the viscosity $\mu$, defined as the ratio of shear stress to shear rate, is given by (Sadowski and Bird, 1965; Savins, 1969; Bird et al., 1987; Carreau et al., 1997):

$\mu=\frac{\mu_{\mathrm{o}}}{1+\left(\frac{\tau}{\tau_{1 / 2}}\right)^{\alpha-1}}$

where $\mu_{0}$ is the low-shear viscosity, $\tau_{1 / 2}$ is the shear stress at which $\mu=\mu_{0} / 2$ and $\alpha$ is an indicial parameter in the model.

From this expression we obtain a formula for the shear rate:

$\gamma=\frac{\tau}{\mu}=\frac{\tau}{\mu_{\mathrm{o}}}\left[1+\left(\frac{\tau}{\tau_{1 / 2}}\right)^{\alpha-1}\right]$

On substituting this into Eq. (13), integrating and simplifying we obtain:

$Q=\frac{\pi R^{3} \tau_{\mathrm{w}}}{4 \mu_{0}}\left[1+\frac{4}{\alpha+3}\left(\frac{\tau_{\mathrm{w}}}{\tau_{1 / 2}}\right)^{\alpha-1}\right]$

where $\tau_{\mathrm{w}}=\Delta P R / 2 L$ and $\Delta P$ is the pressure drop across the tube.
For a Herschel-Bulkley fluid with a yield stress $\tau_{\mathrm{o}}$, a consistency factor $C$ and a behavior index $n$, the shear rate $\gamma$ is given by:

$\gamma=\left(\frac{\tau}{C}-\frac{\tau_{\mathrm{o}}}{C}\right)^{\frac{1}{n}}$

On substituting (17) into (13), integrating and simplifying we get:

$$
\begin{aligned}
Q= & \frac{8 \pi}{C^{\frac{1}{n}}}\left(\frac{L}{\Delta P}\right)^{3}\left(\tau_{\mathrm{w}}-\tau_{\mathrm{o}}\right)^{1+\frac{1}{n}} \\
& \times\left[\frac{\left(\tau_{\mathrm{w}}-\tau_{\mathrm{o}}\right)^{2}}{3+1 / n}+\frac{2 \tau_{\mathrm{o}}\left(\tau_{\mathrm{w}}-\tau_{\mathrm{o}}\right)}{2+1 / n}+\frac{\tau_{\mathrm{o}}^{2}}{1+1 / n}\right] .
\end{aligned}
$$

This relation can also be derived from first principles by considering shear and pressure forces on a cylindrical annulus of fluid to find the velocity profile as a function of radius and then integrating over the cross-section to obtain $Q$.

There are three important special cases for HerschelBulkley fluid. These, with the volumetric flow rate expressions, are:

1. Newtonian

$$
Q=\frac{\pi R^{4} \Delta P}{8 L C}
$$

2. Power-law

$$
Q=\frac{\pi R^{4} \Delta P^{1 / n}}{8 L C^{1 / n}}\left(\frac{4 n}{3 n+1}\right)\left(\frac{2 L}{R}\right)^{1-1 / n}
$$

3. Bingham plastic

$$
Q=\frac{\pi R^{4} \Delta P}{8 L C}=\left[\frac{1}{3}\left(\frac{\tau_{\mathrm{o}}}{\tau_{\mathrm{w}}}\right)^{4}-\frac{4}{3}\left(\frac{\tau_{\mathrm{o}}}{\tau_{\mathrm{w}}}\right)+1\right] .
$$

Each one of these expressions can be derived directly by the two methods presented here. Moreover, they can be obtained by substituting the relevant conditions in the flow rate expression for HerschelBulkley fluid.

\section{Appendix B. Networks}

The physical and statistical properties of the networks are presented in Tables 7 and 8 . 
Table 7

Physical and statistical properties of the sand pack network

\begin{tabular}{ll}
\hline General & \\
\hline Size & $2.5 \mathrm{~mm} \times 2.5 \mathrm{~mm} \times 2.5 \mathrm{~mm}$ \\
Total number of elements & 13,490 \\
Absolute permeability & 93.18 \\
$\quad\left(10^{-12} \mathrm{~m}^{2}\right)$ & \\
Formation factor & 2.58 \\
Porosity & 0.338 \\
Clay bound porosity & 0.00 \\
No. of connections to inlet & 195 \\
No. of connections to outlet & 158 \\
Triangular shaped elements (\%) & 94.69 \\
Square shaped elements (\%) & 1.56 \\
Circular shaped elements (\%) & 3.75 \\
Physically isolated elements (\%) & 0.00
\end{tabular}

Pores

\begin{tabular}{ll}
\hline Total number & 3567 \\
No. of triangular shaped & 3497 \\
No. of square shaped & 70 \\
No. of circular shaped & 0 \\
No. of physically isolated & 0
\end{tabular}

\begin{tabular}{lll} 
& Ave. & St. Dev. \\
\cline { 2 - 3 } Connection number & 5.465 & 4.117 \\
Center to center length $\left(10^{-6} \mathrm{~m}\right)$ & 197.46 & 176.24 \\
Inscribed radius $\left(10^{-6} \mathrm{~m}\right)$ & 39.05 & 15.78 \\
Shape factor & 0.0363 & 0.0081 \\
Volume $\left(10^{-15} \mathrm{~m}^{3}\right)$ & 1062.42 & 2798.79 \\
Clay volume $\left(10^{-15} \mathrm{~m}^{3}\right)$ & 0.00 & 0.00
\end{tabular}

Throats

\begin{tabular}{lll}
\hline Total number & 9923 & \\
No. of triangular shaped & 9277 & \\
No. of square shaped & 140 & \\
No. of circular shaped & 506 & \\
No. of physically isolated & 0 & St. Dev. \\
& & 16.31 \\
& Ave. & 11.19 \\
\cline { 2 - 3 } Length $\left(10^{-6} \mathrm{~m}\right)$ & 30.61 & 0.0137 \\
Inscribed radius $\left(10^{-6} \mathrm{~m}\right)$ & 23.71 & 151.18 \\
Shape factor & 0.0067 & 0.00 \\
Volume $\left(10^{-15} \mathrm{~m}^{3}\right)$ & 155.93 \\
Clay volume $\left(10^{-15} \mathrm{~m}^{3}\right)$ & 0.00 & \\
\hline
\end{tabular}

No. $=$ Number Ave. $=$ Average St. Dev. $=$ Standard Deviation .

\section{References}

Al-Fariss, T.F., 1989. Comput. Chem. Eng. 13 (4/5), 475-482.

Al-Fariss, T.F., Pinder, K.L., 1984. Flow of a Shear-Thinning Liquid with Yield Stress through Porous Media. Paper SPE, 13840.

Alvarado, D.A., Marsden, S.S., 1979. SPE J. 369-377 (December).

Balhoff, M.T., 2004. Modeling the flow of crosslinked guar gum in porous media. Polymer 45 (2), 128-129 (Preprints).

Balhoff, M.T., 2005. Modeling the Flow of Non-Newtonian Fluids in Packed Beds at the Pore Scale, PhD Thesis, Louisiana State University. Balhoff, M.T., Thompson, K.E., 2004. AIChE J. 50 (12), 3034-3048. Balhoff, M.T., Thompson, K.E., 2006. Chem. Eng. Sci. 61, 698-719.
Table 8

Physical and statistical properties of the Berea network

\begin{tabular}{ll}
\hline General & \\
\hline Size & $3.0 \mathrm{~mm} \times 3.0 \mathrm{~mm} \times 3.0 \mathrm{~mm}$ \\
Total number of elements & 38495 \\
Absolute permeability & 2.63 \\
$\quad\left(10^{-12} \mathrm{~m}^{2}\right)$ & \\
Formation factor & 14.33 \\
Porosity & 0.183 \\
Clay bound porosity & 0.057 \\
No. of connections to inlet & 254 \\
No. of connections to outlet & 267 \\
Triangular shaped elements (\%) & 92.26 \\
Square shaped elements (\%) & 6.51 \\
Circular shaped elements (\%) & 1.23 \\
Physically isolated elements (\%) & 0.02
\end{tabular}

Pores

\begin{tabular}{ll}
\hline Total number & 12349 \\
No. of triangular shaped & 11794 \\
No. of square shaped & 534 \\
No. of circular shaped & 21 \\
No. of physically isolated & 6
\end{tabular}

Connection number

Ave. St. Dev.

Center to center length $\left(10^{-6} \mathrm{~m}\right)$ $4.192 \quad 1.497$

48.81

Inscribed radius $\left(10^{-6} \mathrm{~m}\right) \quad 19.17$

Shape factor $\quad 0.0113$

8.47

Volume $\left(10^{-15} \mathrm{~m}^{3}\right)$

300.55

0.0097

Clay volume $\left(10^{-15} \mathrm{~m}^{3}\right) \quad 88.30$

498.53

353.42

Throats

\begin{tabular}{ll}
\hline Total number & 26,146 \\
No. of triangular shaped & 23,722 \\
No. of square shaped & 1972 \\
No. of circular shaped & 452 \\
No. of physically isolated & 3
\end{tabular}

\begin{tabular}{lll}
\cline { 2 - 3 } Length $\left(10^{-6} \mathrm{~m}\right)$ & 13.67 & 5.32 \\
Inscribed radius $\left(10^{-6} \mathrm{~m}\right)$ & 10.97 & 7.03 \\
Shape factor & 0.0022 & 0.0139 \\
Volume $\left(10^{-15} \mathrm{~m}^{3}\right)$ & 48.07 & 43.27 \\
Clay volume $\left(10^{-15} \mathrm{~m}^{3}\right)$ & 17.66 & 34.89 \\
\hline
\end{tabular}

No. $=$ Number Ave.$=$ Average St. Dev. $=$ Standard Deviation .

Bear, J., 1972. Dynamics of Fluids in Porous Media. American Elsevier. Bijeljic, B., Muggeridge, A.H., Blunt, M.J., 2004. Water Resour. Res. 40, W11501.

Bird, R.B., Armstrong, R.C., Hassager, O., 1987. Dynamics of Polymeric Liquids, vol. 1. John Wiley \& Sons.

Blunt, M.J., 2001. Curr. Opin. Colloid Interface Sci. 6 (3), 197-207. Blunt, M.J., Jackson, M.D., Piri, M., Valvatne, P.H., 2002. Adv. Water Resour. 25, 1069-1089.

Cannella, W.J., Huh, C., Seright, R.S., 1988. Proceedings of the 63rd SPE Annual Technical Conference and Exhibition, Houston, Texas. Paper SPE, 18089. 
Carreau, P.J., De Kee, D., Chhabra, R.P., 1997. Rheology of Polymeric Systems. Hanser Publishers.

Chaplain, V., Mills, P., Guiffant, G., Cerasi, P., 1992. J. Phys., II 2, $2145-2158$.

Chase, G.G., Dachavijit, P., 2003. Sep. Sci. Technol. 38 (4), 745-766.

Chen, M., Yortsos, Y.C., Rossen, W.R., 2004. SPE Annual Technical Conference and Exhibition, Houston, Texas. Paper SPE, 90939.

Chen, M., Yortsos, Y.C., Rossen, W.R., 2005a. Colloids Surf., A 256, 181-189.

Chen, M., Rossen, W.R., Yortsos, Y.C., 2005b. Chem. Eng. Sci. 60, 4183-4202.

Dauben, D.I., Menzie, D.E., 1967. J. Pet. Technol. 1065-1073 (August).

Dominguez, J.G., Willhite, G.P., 1977. SPE J. 111-121 (April).

Fadili, A., Tardy, P.M., Pearson, A., 2002. J. Non-Newton. Fluid Mech. 106, 121-146.

Gaitonde, N.Y., Middleman, S., 1966. Symposium on Mechanics of Rheologically Complex Fluids, Houston, Texas. Paper SPE, 1685.

Gücüyener, İ.H., Mehmetoğlu, T., 1996. J. Pet. Sci. Eng. 16, 45-60.

Huang, Y., Sorbie, K.S., 1992. SPE/DOE Enhanced Oil Recovery Symposium, Tulsa, Oklahoma. Paper SPE/DOE, 24153.

Kelessidis, V.C., Maglione, R., Tsamantaki, C., Aspirtakis, Y., 2006. J. Pet. Sci. Eng. 53, 203-224.

Kharabaf, H., Yortsos, Y.C., 1996. Phys. Rev., E 55 (6), 7177-7191.

Kozicki, W., Hsu, C.J., Tru, C., 1967. Chem. Eng. Sci. 22, 487-502.

Lopez, X., Valvatne, P.H., Blunt, M.J., 2003. J. Colloid Interface Sci. 264, 256-265.

Marshall, R.J., Metzner, A.B., 1966. Symposium on Mechanics of Rheologically Complex Fluids, Houston, Texas, USA. Paper SPE, 1687.

Øren, P., Bakke, S., 2003. J. Pet. Sci. Eng. 39, 177-199.

Øren, P.E., Bakke, S., Arntzen, O.J., 1998. Extending Predictive Capabilities to Network Models. Soc. Pet. Eng. J. 3 (4), 324-336.

Park, H.C., 1972. The Flow of Non-Newtonian Fluids Through Porous Media, PhD Thesis, Michigan State University.

Park, H.C., Hawley, M.C., Blanks, R.F., 1973. The Flow of NonNewtonian Solutions Through Packed Beds. Paper SPE, 4722.

Pascal, H., 1981. Acta Mech. 39, 207-224.
Pearson, J.R.A., Tardy, P.M.J., 2002. J. Non-Newton. Fluid Mech. $102,447-473$.

Piri, M., Blunt, M.J., 2005. Phys. Rev., E 71, 026302.

Rossen, W.R., Gauglitz, P.A., 1990. AIChE J. 36, 1176-1188.

Rossen, W.R., Mamun, C.K., 1993. Phys. Rev., B 47, 11815-11825.

Roux, S., Hansen, A., 1987. J. Phys. 20, 1281-1285.

Ruge, J.W., Stüben, K., 1987. In: McCormick, S.F. (Ed.), Multigrid Methods: Chapter 4 (Algebraic Multigrid), Frontiers in Applied Mathematics. SIAM.

Sadowski, T.J., 1963. in: Non-Newtonian flow through porous media, PhD Thesis, University of Wisconsin.

Sadowski, T.J., 1965. Transactions of the Society of Rheology, vol. 9 (2), pp. 251-271.

Sadowski, T.J., Bird, R.B., 1965. Transactions of the Society of Rheology, vol. 9(2), pp. 243-250.

Saffman, P.G., 1959. J. Fluid Mech. 6, 321-349.

Savins, J.G., 1969. Ind. Eng. Chem. 61 (10), 18-47.

Skelland, A.H.P., 1967. Non-Newtonian Flow and Heat Transfer. John Wiley and Sons Inc.

Sorbie, K.S., 1989. SPE Annual Technical Conference and Exhibition, San Antonio, Texas. Paper SPE, 19651.

Sorbie, K.S., 1991. Polymer-Improved Oil Recovery. Blakie and Son Ltd.

Sorbie, K.S., Clifford, P.J., 1989. J. Colloid Interface Sci. 130 (2), 508-534.

Sorbie, K.S., Clifford, P.J., 1991. Chem. Eng. Sci. 46 (10), 2525-2542.

Sorbie, K.S., Parker, A., Clifford, P.J., 1987. SPE Reserv. Eng. 281-304 (August).

Tsakiroglou, C.D., 2002. J. Non-Newton. Fluid Mech. 105, 79-101.

Tsakiroglou, C.D., 2004. J. Non-Newton. Fluid Mech. 117, 1-23.

Tsakiroglou, C.D., Theodoropoulou, M., Karoutsos, V., Papanicolaou, D., Sygouni, V., 2003. J. Colloid Interface Sci. 267, 217-232.

Valvatne, P.H., Blunt, M.J., 2004. Water Resour. Res. 40, W07406.

Vradis, G.C., Protopapas, A.L., 1993. J. Hydraul. Eng. 119 (1), 95-108.

White, D.A., 1967. Chem. Eng. Sci. 22, 669-672.

Wu, Y.S., Pruess, K., Witherspoon, P.A., 1992. SPE Reserv. Eng. 369-376 (August). 